\title{
Coupling of Piezoelectric Effect with Electrochemical Processes
}

\author{
Matthew B Starr, Xudong Wang* \\ Department of Materials Science and Engineering, University of Wisconsin-Madison \\ Madison, WI 53706, USA \\ Email: xudong@engr.wisc.edu
}

\begin{abstract}
The coupling effect between piezoelectric polarization and electrochemical processes depicts the engineering of charge-carrier conduction characteristics at the heterojunction between a strained piezoelectric material and a chemical solution. It is a unique subcategory of piezotronics. This mini review paper introduces the fundamental principles of such coupling effects. Applications of this coupling effect are reviewed and discussed in several different aspects, including selective etching enabled by piezo(ferro)-electric polarization; selective (photo)electrochemical deposition directed by piezo(ferro)-electric potential; and the directly utilization of piezoelectric potential to drive electrochemical reactions (piezocatalysis). At the end, perspectives of this coupling effect are discussed as a new approach in the fields of corrosion management, nanomanufacturing and renewable energy conversion.
\end{abstract}

Key words: Piezotronics, Piezoelectric, Ferroelectric, Piezocatalysis, Electrochemistry

\section{Introduction}

Piezoelectrics are a class of materials that create an electric field in response to mechanical deformation. The origin of the electric field (i.e. piezoelectric field) is a break in the inversion symmetry which lead to electric dipoles within the material, the strength of which changes as the material is strained. The changing dipole field leads to a change in the electric field which permeates the material (Figure 1). Piezoelectrics as a phenomenological description encompasses 
three subcategories of materials: ferroelectrics, pyroelectrics and piezoelectrics (Figure 2) ${ }^{1,2}$. Piezoelectric materials that are non-ferroelectric and non-piezoelectric (e.g. $\mathrm{SiO}_{4}$ ) have no electric dipoles when under no strain, but upon strain there is a separation between the positive and negative charge centers which results in an non-zero electric field. Pyroelectric materials that are non-ferroelectric (e.g. $\mathrm{LiTaO}_{3}$ ) exhibit spontaneous polarization: even in the absence of mechanical deformation or an electric field, the positive and negative charge centers in each unit cell do not coincide, giving rise to a resultant electric dipole. Upon strain, the separation between charge centers changes and thus the electric field changes. Pyroelectric materials will also experience a change to their dipoles when subjected to a change in temperature. The polarization in piezoelectric materials that are not ferroelectric is not permanently changeable by applying an external electrical field. Ferroelectric materials (all of which are piezoelectric) also contains a spontaneous polarization in the absence of strain, as well as a temperature-sensitive polarization, but differ from the other classes of materials in that a ferroelectric material's spontaneous dipoles need not necessarily align from one unit cell to the next. Regions in which neighboring dipoles are aligned with one another are called domains. The direction of spontaneous polarization in a ferroelectric's unit cell can be changed if exposed to a sufficiently strong electric field.

The electric field exhibited by piezoelectric materials, whether strain induced, temperature induced, or spontaneously realized, can have a dramatic effect on the electronic properties both inside and outside of the material. Electric fields disrupt the energy of electronic states throughout the material (Figure 3a) and can cause the rearrangement of free charge inside and outside of the material (Figure 3b). This electronic rearrangement can dramatically affect chargecarrier conduction characteristics at the heterojunction between a piezoelectric material and another medium, because these characteristics depend sensitively upon the continuity of occupiable electronic states from one medium to the other and on the number of charges free to move between the mediums. This phenomenon is known as the piezotronics effect ${ }^{3-5}$. One such heterojunction that is particularly dynamic is that which exists between a piezoelectric material and a chemical solution ${ }^{6,7}$. Piezoelectric fields have been used to control the corrosion rate of materials exposed to etchant solutions ${ }^{8-18}$, selectively control the energetics and spatial separation of adsorbed $^{19,20}$ and photo-deposition materials ${ }^{21-28}$, and have even been used to directly drive electrochemical reactions across piezoelectric/solution interface ${ }^{6,7,29-31}$. 
Using piezoelectric fields to influence chemical reactions happening at the material's surface is closely related to electrochemical processes which typically take place at the surface of an electrode in contact with solution, influenced by an outside voltage source (Figure 4). A chemically stable electrode material in contact with solution containing electroactive species will arrive at a steady state electrochemical equilibrium between the electrode and solution (Figure 4a). Applying a voltage to a metal electrode can have one of two results: (1) the electrode potential rises above that of unoccupied states in solution, or (2) the electrode potential drops below that of occupied states in solution. In the first case it is now energetically favorable for there to be a net electron flow from occupied states in the electrode to unoccupied states of electroactive species in solution (i.e. cathodic current, Figure 4b). In the second case it is energetically favorable for a net flow of electrons to come from occupied states of species in solution into the unoccupied states in the electrode (i.e. anodic current, Figure 4c). In the case of an insulating piezoelectric material (Figure 4d) the piezoelectric field, induced by mechanically deforming the piezoelectric material, exerts an energy shift on the occupied (valence band) and unoccupied (conduction band) states throughout the material. With sufficient shifts in the energies of these states, it is possible to drive the flow of electrons from the piezoelectric's surface to species in solution or from species in solution to the piezoelectric's surface (Figure $4 \mathrm{e})^{7}$. In pyroelectric and ferroelectric materials, the presence of a spontaneously dipole means an electric field, and its associated electronic distortion, is present throughout the material even in the absence of an applied strain. It is the presence of these spontaneous dipoles that allows

pyroelectric and ferroelectric materials to exhibit useful corrosion, photochemical and electrochemical properties.

\section{Applications of the Piezopotential-Electrochemistry Coupling Effect}

\subsection{Piezopotential used for material removal}

The selective removal of a material can have disastrous effects, such as in the case of corrosion, or being used to craft useful, precisely engineered structures like undercut etching in 
microelectromechanical (MEM) or nanoelectromechanical (NEM) device fabrication ${ }^{32-41}$. Regardless whether the process is purely chemical (electro-less) dissolution (Figure 5a), electrochemical (Figure 5b) or photoelectrochemical etching (Figure 5c), the selective removal of material is a two-step process:

1) The bonds that hold an atom to the surface of the material are broken. In solid state materials like semiconductors, the valence band represents the energy states of the electrons bonding the atoms of the material together. A broken bond is represented as a hole and thus processes that create holes (e.g. thermal or photoexcitation, electron extraction, etc.) or increase the concentration of holes (e.g. inversion regions) weaken the cohesive forces holding the materials constituent parts together.

2) The saturation of a dangling atom by nucleophilic species in solution (e.g. $\mathrm{OH}^{-}$). When the bonds holding an atom to the surface of a material are broken, the atom is susceptible to having its broken bonds satisfied either by nucleophilic species from solution, that can then surround and shepherd the dangling atom away from the surface, or by an native electron from elsewhere in the material (and thus mending the material).

The electric dipoles present in piezoelectric materials influence the rate of dissolution by acting to enhance process 1 . The electric field present within the material applies a force to all of the material's charges, causing segregation of broken (holes) and unbroken bonds to opposite sides of the material. The side of the material that accumulates the broken bonds is more damaged, experiences more dangling atoms and is more susceptible to dissolution. In addition to concentrating broken bonds, the piezoelectric field also shifts the relative energies of occupied and unoccupied states across the material. Where the piezoelectric field increases the energy of electronic states occupied by electrons, the thermodynamic driving force for electron extraction by electroactive species in solution is increased and thus the number of broken bonds is increased, making more opportunities for nucleophilic attack.

The precise manufacturing of fine ferroelectric structures has found use in MEM devices ${ }^{32-40}$, NEM devices ${ }^{41}$, non-volatile memories ${ }^{42-44}$ and integrated optics ${ }^{13}, 16,17$. Conventionally, piezoelectric elements have been processed by chemical wet etching or argon milling techniques. 
In ferroelectric materials, anisotropic etching is widely observed between regions of differing

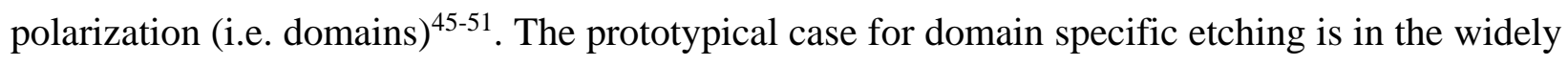
studied optoelectronic material, ferroelectric $\mathrm{LiNbO}_{3}$, etched by a $\mathrm{HF}: \mathrm{HNO}_{3}$ solution ${ }^{52}$. Figure 6 is a schematic showing what one would expect to see if a free-standing $\mathrm{LiNbO}_{3}$ film with a periodic domain structure, as is typically used in optoelectronic applications, were exposed to an etchant solution. In $\mathrm{LiNbO}_{3}$, negative domains (areas with a negative surface charge) etch appreciable faster than positive domains.

$\mathrm{LibNbO}_{3}$ is a wide bandgap material that has negligible free charge carriers. The different etching rates observed on the positive and negative domain surfaces are due to the energetic difference between the electroactive etchant species in solution and the valence band position on the negative and positive domain's surface. At the negative domain's surface, the energy of occupied electronic states is elevated, making them more easily accessible to electron extraction by etchant species. There is a reversed effect on the positive domain's surface, where the energy of occupied electronic states is reduced with respect to the unoccupied states that characterize etchant species.

\subsection{Piezopotential used for selective deposition}

The piezoelectric field's augmentation of electronic states can be wielded to enable the selective growth of new material on the pieozelectric's surface. The adhesive forces that define the bond between the new material and the pieozelectric's surface come in two forms, secondary bonding and primary bonding. Secondary bonds are defined by their weak cohesive forces, e.g. electrostatic and van der Waals bonds. Material additions made to the piezoelectric that have this bonding character may subsequently experience a desorption process with an energy barrier dependent on piezoelectric potential. Primary bonds experience a strong ionic or covalent character. These strong inter-material bonds mean they experience intimate electrical and mechanical relationships with the piezoelectric and may readily be used to enhance or inhibit one or more of the piezoelectric's native surface properties. 


\subsubsection{Effect of polarization on absorption and desorption energy of polar and nonpolar molecules}

Electrostatic and van der Waals forces can characterize the attractive forces bonding polar and nonpolar molecules to piezoelectric's surface ${ }^{53-58}$. Experiments carried out by Altman and Yun evaluated the influence of ferroelectric domain polarization on the absorption and desorption energy of polar acetic acid and 2-propanol and nonpolar dodecane on the (0001) surface of ferroelectric $\mathrm{LiNbO}_{3}{ }^{19}$. Careful absorption and desorption cycles were carried out in an ultrahigh vacuum (UHV) chamber equipped with a differentially pumped microwave electron cyclotron resonance plasma source, an X-ray source, a cylindrical mirror analyzer, and a quadrupole mass spectrometer. The two polar molecules were found to adsorb significantly more strongly on the positive surface (Figures 7a, b). Temperature-programmed desorption (TPD) data yielded desorption energies of the two polar molecules more than 11 orders of magnitude lower than expected. The non-polar dodecane adsorption/ desorption desorption energies were independent of surface polarity (Figures 7c, d).

Absorption and desorption experiments were conducted at multiple temperatures. These experiments demonstrated a dependence of the polar molecules' desorption energies on temperature. Altman and Yun analyzed the trend of desorption with temperature and determined the difference to be due to the pyroelectric property of $\mathrm{LiNbO}_{3}$, where the spontaneous dipole (and the corresponding surface charge) change as a function of temperature. In the end, electrostatic interaction between the pyroelectric surface and the polar molecules were shown to effect surface absorption properties, encourage or discourage desorption of surface molecules.

\subsubsection{Piezopotential-engineered photo deposition}

The coincidence of piezoelectric domain structures being on the order of nanometers and the persistent interest in the bottom-up manufacturing of miniscule electrical components for integrated circuit, memory and sensing applications has encouraged the investigation of how piezoelectric potentials, and in particular ferroelectric domain structure engineering, can be used to control the atomic deposition and growth of new materials. In this instance, the ferroelectric 
polarization acts as a kind of 'mask', dictating the locations of material deposition and growth. A number of experiments have been carried out on both ferroelectric particles and films to explore the feasibility of this possibility ${ }^{21-28}$. Bulk ferroelectric materials exhibit a complicated arrangement of spontaneous electrical polarization orientations. In small ferroelectric particles, it is often observed that a single polarization orientation (domain) dominates the entire particle (Figure 8$)^{59}$. Because of their simple dipole arrangement, ferroelectric particles make an ideal system for testing polarization's influence on material deposition processes.

Studies conducted by Rohrer and Giocondi on ferroelectric $\mathrm{BaTiO}_{3}$ particles less than 5 microns in diameter demonstrated the ability of the spontaneous polarization within these particles to spatially segregate photodeposition reactions occurring on their surfaces (Figure 9). In a series of experiments, $\mathrm{BaTiO}_{3}$ particles were suspended in an aqueous solution containing $0.115 \mathrm{M}$ aqueous $\mathrm{AgNO}_{3}$ (for the oxidation reaction) and $0.0115 \mathrm{M}$ aqueous $\mathrm{Pb}\left(\mathrm{C}_{2} \mathrm{H}_{3} \mathrm{O}_{2}\right.$ ) (for the

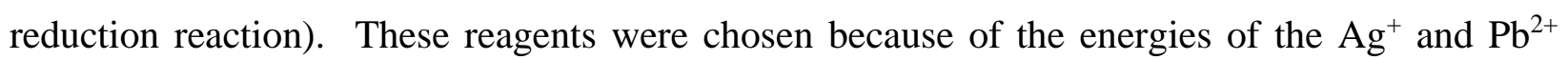
redox potentials, which are within BaTiO3's bandgap (Figure 10).

The reactions expected to occur on opposite facets of the particles are as follows:

$$
\begin{aligned}
& \mathrm{Ag}^{+}+\mathrm{e}^{-}=\mathrm{Ag}, \\
& \mathrm{Pb}^{2+}+2 \mathrm{H}_{2} \mathrm{O}+2 \mathrm{~h}^{+}=\mathrm{PbO}_{2}+4 \mathrm{H}^{+} .
\end{aligned}
$$

The $\mathrm{BaTiO}_{3}$ solution was continuously stirred and exposed to a UV lamp capable of photoexciting charges within the $\mathrm{BaTiO}_{3}$ particles. Careful investigation of the particles' surfaces after UV exposure revealed that the particles photochemically reacted with the electroactive species in their surroundings. Evidence for lead oxidation was found on the ${ }^{60},\{110\}$, and $\{111\}$ surfaces. Silver reduction was most prominently found on the $\{001\}$ surface. Giocondi et al attribute the photodeposition selectivity of the $\mathrm{BaTiO}_{3}$ particles to their spontaneous polarization. The spontaneous polarization is theorized to influence photodeposition of solid $\mathrm{Ag}$ and $\mathrm{PbO}$ by controlling the migration direction of photoexcited electrons and broken bonds (holes) in the particles (Figure 11). 
In the large band gap material $\mathrm{BaTiO}_{3}$, photoexcited electrons occupy conduction band states that are at higher potential energy than the reduction potential of the electroactive $\mathrm{Ag}^{+}$cations in solution. These energetic, photoexcited electrons can lower the systems energy by transferring to the electroactive cations, in this case reducing them from $\mathrm{Ag}^{+}$to solid $\mathrm{Ag}$ metal. On the other end of the particle, broken bonds (holes in the valence band) accumulate and are able to accept electron injection from any number of courses, including water moles.

The ability of spontaneous dipoles to orchestrate the photodeposition process was further investigated by Zhang, Dunn et $\mathrm{al}^{23,26}$. In a series of carefully conducted experiments, Q. Zhang and Dunn showed that for ferroelectric lead zirconate titanate $\left(\mathrm{PbZr}_{\mathrm{x}} \mathrm{Ti}_{1-\mathrm{x}}\right) \mathrm{O}_{3}$ films, photoreduction and photoxidation reactions occur nearly exclusively on the positive and negative domain surfaces, respectively (Figure 12). Zhang and Dunn were able to show that poling the ferroelectric with an AFM tip is capable of engineering the shape of photodeposited structures. Checkerboard patterns, nested squares and wire shapes were all poled in the surface of a PZT film and shown to predictably control the photodeposition of structures on its surface.

Subsequent work by Rohrer et al examined the influence of ferroelectric dipoles on photochemical processes occurring beyond the ferroelectric/solution interface ${ }^{61}$, ${ }^{2}$. Rohrer conducted a series of studies to examine how the ferroelectric dipoles effected the photochemical reactivity of $\mathrm{TiO}_{2}$ films of varying thicknesses deposited by by pulsed laser deposition on the surface ferroelectric $\mathrm{BaTiO}_{3}$. The influence of spontaneous polarization was indeed seen in the patterns of photodeposited $\mathrm{Ag}$ on the $\mathrm{TiO}_{2}$ surface, even when the $\mathrm{TiO}_{2}$ film thickness extended 100nm away from the buried ferroelectric's surface (Figure 13). A careful set of characterization experiments was able to confirm that it was the domain polarization alone, and not other surface features correlated with polarization, which determined the photodeposition behavior of the $\mathrm{TiO} 2$ coating.

\subsection{Piezopotential-Driven Materials Evolution}

In the subsequent section we reviewed how the dipoles present in piezoelectric materials could influence material dissolution, the condensation and evaporation of a gas phase, and the 
photodeposition of an electro active species in solution. The same process that physically segregates reduction and oxidation photodeposition reactions can also be used to separate concurrent gas evolution reactions, such as the photolysis of water into $\mathrm{H}_{2}$ and $\mathrm{O}_{2}$, where back reactions can be parasitic to the energy conversion process.

\subsubsection{Piezopotential-engineered photoelectrochemical material evolution}

The conversion of renewable energy sources, especially solar, into chemical fuels that can provide drop-in, sustainable drivers for our energy intensive economy has been a scientific pursuit for decades ${ }^{63-69}$. One of the many ensembles currently pursued for achieving this goal is the 'photocatalytic' approach, whereby a semiconductor or a series of semiconductor heterojunctions absorbs light from the sun and uses the photoexcited carriers to simultaneously electrochemically reduce and oxidize water to $\mathrm{H}_{2}$ and $\mathrm{O}_{2}$ gas, respectively ${ }^{66,69-88}$. The $\mathrm{H}_{2}$ and $\mathrm{O}_{2}$ gas can then be extracted and used by fuel cells to generate electricity, or the $\mathrm{H}_{2}$ can be used to synthesize carbon based fuels by combining it with reduced $\mathrm{CO}_{2}$. Many designs have been proposed for the photocatalytic approach. One of the economically inexpensive of the proposed approaches consists of a suspension of light sensitive semiconductor particles in a transparently encapsulated pool of water where the particles have direct access to both the sunlight and $\mathrm{H}_{2} \mathrm{O}$ (Figure 14). This approach has encountered a number of challenges, including a high recombination rate of photoexcited charges within the semiconductor particles themselves and the back reaction of $2 \mathrm{H}_{2}$ and $\mathrm{O}_{2}$ molecules into $2 \mathrm{H}_{2} \mathrm{O}$ in the moments immediately following the electrochemical splitting of water on the particles surface.

Semiconducting ferroelectric particles employed for the photolysis of $\mathrm{H}_{2} \mathrm{O}$ offers an eloquent solution to both of these challenges ${ }^{89-94}$. In small particles, electrons and holes are spatially confined resulting in a high rate of photoexcited charge recombination. The spontaneously polarization present throughout a ferroelectric particle pushes the electrons and holes to opposite sides of the material, reducing their wavefunction overlap and thus reducing their probability of recombination. A reduced recombination rate leads to a longer carrier lifetime and subsequently a greater number of attempts for the photoexcited electron and hole to react with their chemical 
environment. In addition to reducing the recombination rate and increasing the likelihood that a photoexcited carrier will react with its chemical environment, the segregation of electrons and holes also ensures that the electrochemical reduction and oxidation of water will happen on spatially segregated surfaces. The separation of reaction products $\left(\mathrm{H}_{2}\right.$ and $\mathrm{O}_{2}$ in this case) dramatically reduces the likelihood that these chemical species will immediately back-react to form water.

\subsubsection{Piezopotential-Driven Electrochemistry}

The use of piezoelectric materials in chemical systems was classically restricted to their 'static' deployment. In these cases, the piezoelectric would be un-poled, poled, or statically strained and the resulting spontaneous or strain-induced piezoelectric field would modify the energetics of electric states and the direction of migrating changes, to achieve some engineered goal ${ }^{60,89-95}$. Recently there have been investigations into the use of dynamic applications, where a piezoelectric material is continuously and alternately strained between a tensile and compressive state resulting in a large and rapidly varying piezoelectric field within the material. This continuously varying system is prevented from achieving thermodynamic (electrochemical) equilibrium with its environment, the result of which is an enduring, though fluctuating, exchange of charge between the piezoelectric and its environment ${ }^{6,7,29,30}$.

The procedure of a typical electrochemistry experiment includes submerging an electrode of interest in a chemical environment of interest and then using an external power source to control the flow of charge between the electrode/environment interface. Li et al explored a set of experiments that combined the power source and electrode into a single material ${ }^{29,30}$. Using an ultrasonic bath and water based solutions, $\mathrm{Li}$ subjected suspensions of wire-like $\mathrm{ZnO}$ and $\mathrm{BaTiO}_{3}$ to ultrasonic vibration and micro-cavitation forces with the objective of getting them to bend and strain, generate a piezoelectric field, and piezoelectrochemically split water into $\mathrm{H}_{2}$ and $\mathrm{O}_{2}$. While a number of questions remain as to what kinds of forces the piezoelectrics were subjected to and what magnitude of piezoelectric potentials were created, the $\mathrm{H}_{2}$ and $\mathrm{O}_{2}$ evolution as a function of piezoelectric ultrasonication were clearly correlated (Figure 15). 


\subsubsection{Piezocatalysis on metal surfaces}

Three factors complicated a detailed investigation in $\mathrm{Li}$ et al.'s system, including: 1) the logistical difficulties with observing nanomaterials in situ, 2) the known unknowns surrounding the surface structure and chemistry of nanomaterials, and 3) the miniscule signal that is expected to come from any single nanostructure even under ideal circumstances due to piezoelectric materials low surface charge density combined with a nanomaterials low surface area. Given these limitations, Starr et al. further investigated the use of piezoelectric potential as a power source for driving electrochemical reactions by using a bulk $(24 \mathrm{~mm} \times 4 \mathrm{~mm} \times 0.25 \mathrm{~mm})$ single crystal of piezoelectric PMN-PT, coated with gold electrodes, attached to a computer controlled actuator for applying strain (Figure 16) ${ }^{6}$.

Starr's experiments showed a correlation between the systems $\mathrm{H}_{2}$ output (a byproduct of the $\mathrm{H}_{2} \mathrm{O}$ reduction reaction) with both the amplitude and frequency of strain subjected to the piezoelectric (Figure 17). Additional experiments to study the effect of electrolyte concentration on the efficiency of the mechanical to chemical energy conversion process revealed a dramatic inverse relationship (Figure 18). In piezoelectrochemistry systems, the electrolyte's spectator ions contribute to a detrimental capacitive current, sapping the electrical potential energy stored in the piezoelectric and thereby interfering with the conversation of piezoelectric surface charge into chemical energy.

The results gained from the bulk PMN-PT experiment were later generalized to other piezoelectric materials and the hydrogen production capacity of those materials was calculated as a function of their unique material properties (Figure 19) ${ }^{7}$.

\subsubsection{Piezocatalysis on bare piezoelectric surfaces}

Starr et al. theoretically extend their experimental results to a host of other piezoelectric materials, each with their own mechanical, electrical and piezoelectric properties ${ }^{7}$. Select efficiency parameters were extracted from their experimental work and applied to bare 
piezoelectrics. The individual ability of the bare piezoelectrics to drive the evolution of $\mathrm{H}_{2}$ as a function of mechanical strain was then compared (Figure 20).

At high strains, the plots of the mechanical-to-chemical $\left(\mathrm{H}_{2}\right)$ production capacity for metal coated and bare piezoelectric materials behave similar. In both instances a high strain generates a strong piezoelectric field, the effects of which are much stronger than the differences seen between the bare piezoelectrics and the metal coated materials, namely the electronic state occupancy about the Fermi energy. At small strains, the similarity seen in $\mathrm{H}_{2}$ production capacity (Insert of Figure 20) is superficial. Qualitatively and quantitatively, the electrochemical behavior of bare piezoelectric materials differs from that of metal coated piezoelectrics. In Wang's model, the difference between a metal coated PZT material and a bare PZT slab arises from the difference between the Fermi energy of the metal and the conduction and valence band states of the bare piezoelectrics. In metals, there are a large number of occupied and unoccupied states about the Fermi energy for donating and accepting electrons, respectively, whereas an ideal semiconductor can only accept additional electrons in its conduction band and donate electrons from its valence band. Unlike in a metal, semiconductors possess an energy gap between these acceptor and donor states which the piezoelectric potential must move up or down in energetic space in order to initiate an electrochemical reaction (Figure 21).

\section{Perspective}

As long as electrical charges play a role in the removal, addition, or evolution of chemical species from the surfaces of a piezoelectric, an electric field inside the piezoelectric will affect the directionality and locality of these chemical processes. Photoexcited charges are one way of driving chemical reactions, and the dipoles in unstrained ferroelectrics have been used to direct their flow. A piezoelectric that is non-ferroelectric can be strained to induce dipoles that can be used to influence photoexcited charges. However, these materials can never be poled like ferroelectrics and thus the spatial locality of chemical reactions cannot be intricately written into their surface, which is a useful property when making a polarization 'mask' for depositing intricate micro and nanostructures. 
Some instances require strain in order to drive chemical reactions between the piezoelectric and the environment. For example, when a piezoelectric is in equilibrium with its surroundings and there is no illumination to provide photoexcited charges to drive chemical reactions, then an electronic perturbation, such as that which accompanies the mechanical strain of a piezoelectric, is required to push or pull electrons out of or into the surface of the piezoelectric.

Much of the work done to date for exploring the use of piezoelectric polarization for influencing chemical reactions at the surface of the piezoelectric, or within the vicinity of the piezoelectric, have focused on simple system and delivered proof of principle results. Extending the work of polarization's effect on corrosion rate beyond the development of a characterization technique for confirming domain structure orientation, for example to applications in corrosion prevention, is a natural but meaningful progression of this work. Rohrer's work with ferroelectric BTO coated with $\mathrm{TiO}_{2}$ films demonstrates that the effect of ferroelectric polarization can extend well beyond the piezoelectric's surface, affecting the chemical reactivity of its surroundings. This work points the way to developing paints and other coating materials impregnated with polarized piezoelectric materials that imbue the objects they coat with enhanced corrosion resistance properties.

The selectively photo deposition of precise nanostructures on specific ferroelectric domains may have important applications for device manufacturing if several difficulties can be overcome. In practice, an AFM can be used to 'write' extremely small and intricate polarization structures on the surface of a ferroelectric, and the use of photochemistry triggered by broad UV exposure opens up the possibility of large area, simultaneous growth of photodeposited nanostructures across an entire wafer. However, the AFM writing process is slow, akin to the slow and expensive process of using electron beams to make fine-featured photolithography masks. Like in current photolithographic schemas, the ferroelectric polarization 'masks' made by AFM will be expensive and viewed as a non-consumable. Today, photodeposition of nanostructures on written ferroelectric domains proceeds directly on the surface of the ferroelectric, with no regard to how these structures could be securely removed and transferred without irreversibly damaging the expensive ferroelectric mask. A possibility forward is to focus work on developing film coatings for the ferroelectric masks that will be strongly photochemically influenced by the 
ferroelectric domains (such as in Rohrer's work with $\mathrm{TiO}_{2}$ films) and may also be easily removed as a sacrificial layer, making transferability of the photochemically deposited nanostructures possible. Following the nanostructure transfer step, the process could again be repeated with a new sacrificial film being grown on top of the ferroelectric mask and the photodeposition step proceeding. Realizing a process like this means the expensive ferroelectric polarization mask could be used many times, reducing the cost of using this strategy for nanoarchitecture growth.

The use of ferroelectric particles as a single material system for simultaneously 1) absorbing solar energy, 2) separating photoexcited electrons and holes to reduce charge recombination, and 3) spatially segregating reaction products and thereby reducing the probability of back reactions, is a tantalizing prospect. Additional measures are needed to overcome these obstacles when using non-ferroelectric material systems, such as binary phase particles and artificial membranes to help separate photoexcited charges and reaction products. Ferroelectric water photolysis systems still face many challenges, such as large bandgaps and low activity with respect to water splitting, but a renewed interest in perovskite light absorbers that has recently taken to the literature and will no doubt be accompanied by the creation of novel ferroelectric materials for future photolysis systems to exploit.

When applying the direct piezoelectric effect as the driving force for electrochemical reactions on bare piezoelectrics, whether it be a nanomaterial's facet or a bulk slab's polished surface, the biggest questions that remain pertain to what exactly is the energetic effect of the piezoelectric field on the surface electronic states and what is the exact nature of the interaction between electroactive species in solution and the piezoelectrics' surface atoms. These systems have promise, directly leveraging the ubiquity of mechanical energy for use in energy storage or environmental chemical remediation applications. The presence of metal films on the piezoelectric's surfaces greatly reduces the complexity of these systems, and perhaps makes the systems immediately suited for some applications, but these films also obscure our ability to observe novel phenomena in this unexplored field. If we don't know how the systems work, we cannot intelligently engineer better systems. 
Until recently it would be impossible to probe the electronic nature of the surface of these piezoelectric materials, under strain, while exposed to various solution chemistry. Developments in the field of ambient pressure x-ray photoelectron spectroscopy (AP-XPS) are beginning to shine light on once unseen electronical and chemical phenomena occurring in-situ in fuel cells during their operation. These new AP-XPS techniques can be extended to strained piezoelectric systems, where the electronic evolution of a piezoelectric surface can be observed in real-time, during mechanical strain and exposed to a condensed liquid phase. These results can feedback in the materials design process, focusing on elemental compositions that most readily react with desired electroactive species without going as far as to irreversibly deconstruct the piezoelectrics bonds.

\section{Conclusion}

The ability of piezoelectric materials to create an electrical polarization, either spontaneously (e.g. pyroelectrics and ferroelectrics) or through mechanical deformation (i.e. direct piezoelectric effect), has been traditionally been exploited for mechanical actuators and radar systems where they mediate the conversion of electrical signals into mechanical signals. As our use of technology has evolved, we seek novel ways of engineering on ever-finer scales and require new means of converting the ubiquitous, renewable energy around us into the electrical and chemical forms that our technology demands. Piezoelectric materials have again and again demonstrated their ability to do more with less by allowing us to tailor the mechanical, electrical, and now chemical properties simultaneously within a single material, for specific applications. Corrosion management, nanomaterials growth and renewable energy conversation, from both mechanical and solar means to both electrical and chemical ends, are all fields that stand to benefit from further investigation of the novel interactions between piezoelectric materials and their chemical environment.

\section{Acknowledgements}


The authors thank the supported by National Science Foundation under award number CMMI1148919.

\section{Authors Bio:}

Mr. Matthew Starr is a graduate student in Materials Science Program at the University of Wisconsin-Madison under the advisement of Prof. Xudong Wang. He received his B.Sc in Materials Science and Engineering at Rutgers University in 2009. He enjoys teaching undergraduate materials science courses and diving deep into conversations that question the basic assumptions that underlie well accepted heuristics. When he's not throwing together custom equipment for one-of-a-kind experiment or simulating future experiments using Mathematica, he enjoys designing and building consumer product prototypes. His current research focuses on illuminating the interaction between piezoelectricity and electrochemistry.

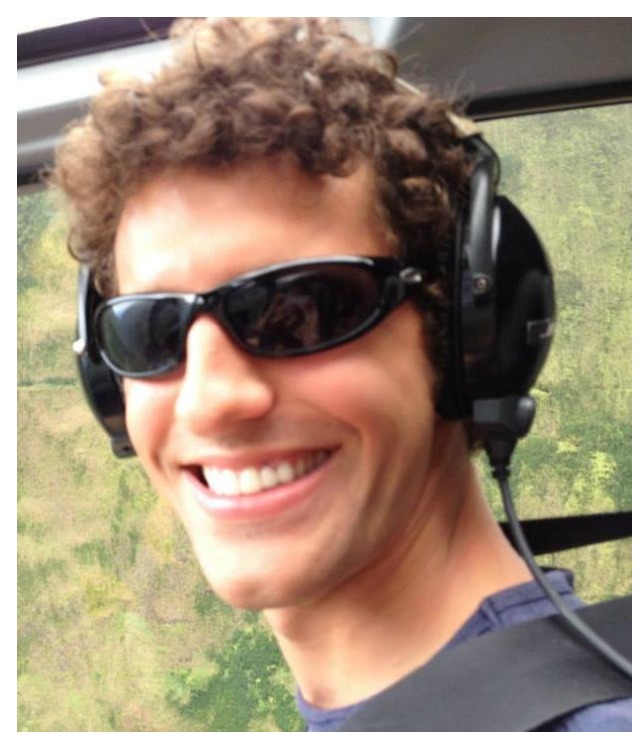

Dr. Xudong Wang is an associate professor in the department of Materials Science and Engineering at University of Wisconsin-Madison. His research interests include studying the growth and assembly of oxide nanowire arrays, understanding the coupling effect of semiconductor properties and piezoelectric charge displacement, and developing nanogenerator that uses piezoelectric nanomaterials to convert low level mechanical energy into electricity. He has published more than 70 papers in peer reviewed scientific journals, contributed 7 book chapters, and holds 7 patents/provisional patents. His publications have been cited over 7,000 times by peers and his current h-index is 34 . 


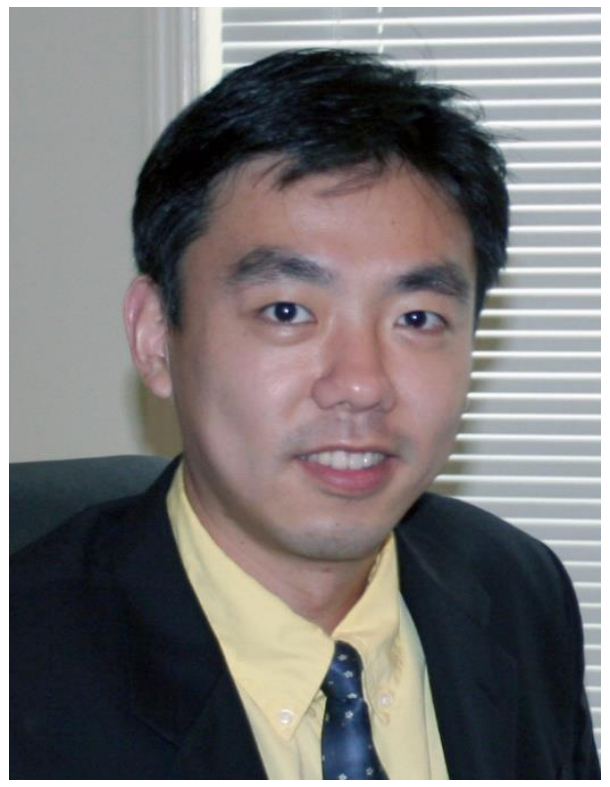


Figures and Figure Captions

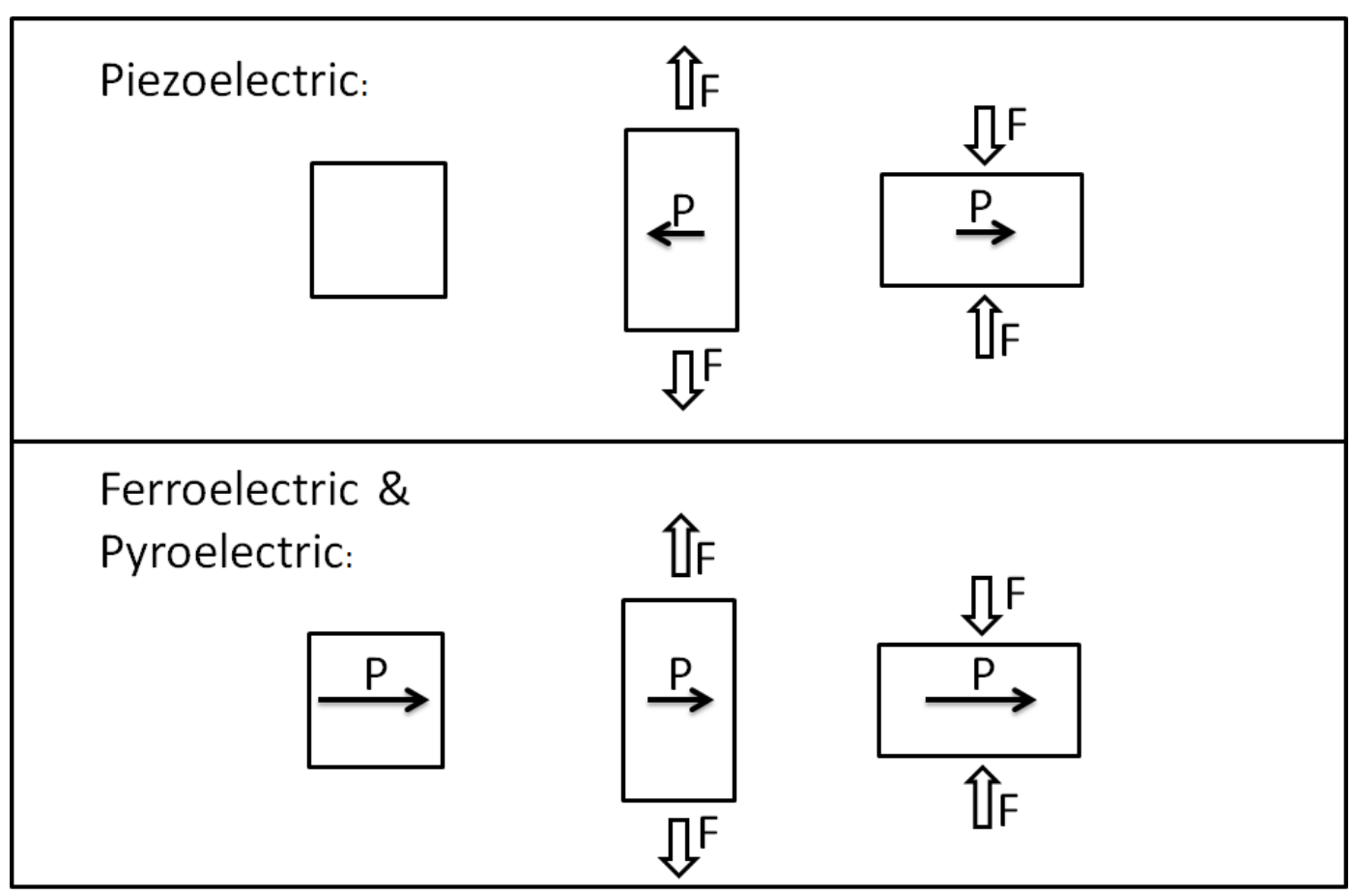

Figure 1. a) Piezoelectric materials that are non-ferroelectric and non-pyroelectric being with zero internal dipole. When these materials are strained, the lattice displacement creates dipole moments that create an electric field across the material. b) Ferroelectric and pyroelectric materials have spontanious dipoles without strain. When these materials are strained, their dipoles change in strength, again changing the electric field saturating the material. 


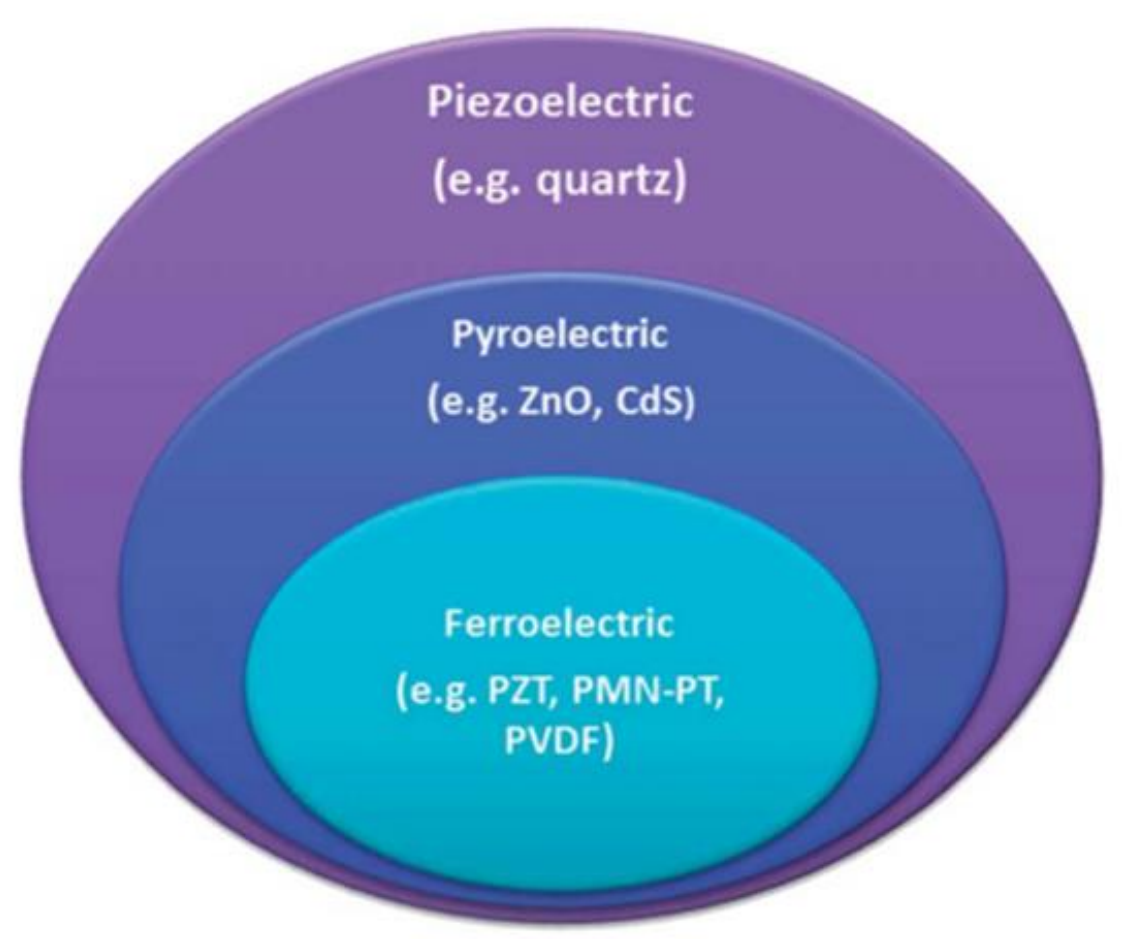

Figure 2. Piezoelectric materials experience a change in internal dipole moment when mechanically deformed. Pyroelectrics are kind of piezoelectric material which have spontaneous dipoles in the absence of mechanical deformation. The dipoles cannot be made to permanently switch direction by applying an external electric field. The spontaneous dipoles of pyroelectric materials changes as a function of temperature. Ferroelectric materials are a special subclass of pyroelectrics. The spontaneous dipole in ferroelectric materials can be made to permanently change orientation by applying a strong enough external electric field. 


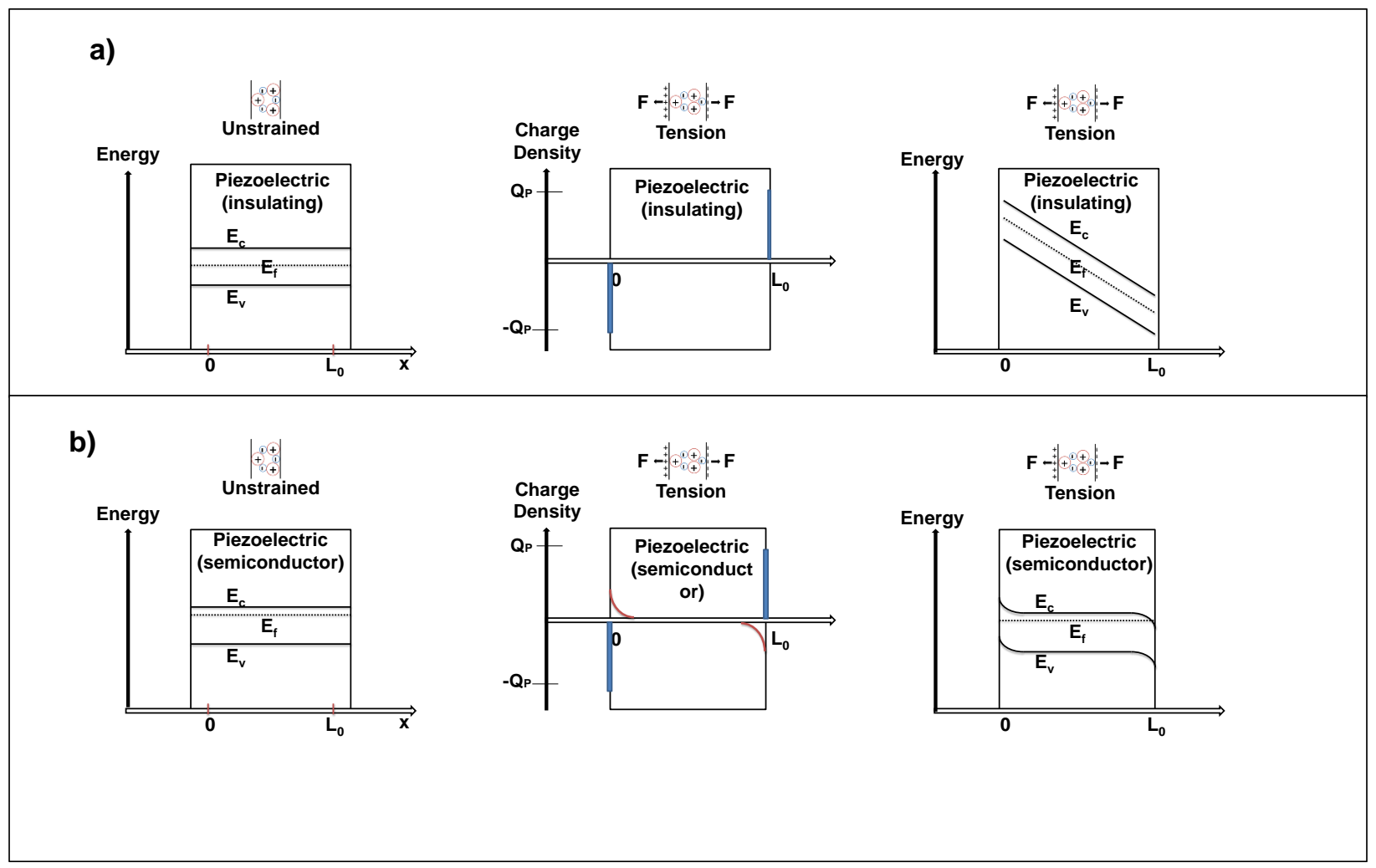

Figure 3. The electric field created by straining a piezoelectric material effects the energetics of electronic states throughout the material. a) in a perfect insulator, no charge is able to move in response to the piezoelectric field and the electric field linearly augments the energy of occupied (valence band) and unoccupied (conduction band) states across the material. b) In a semiconducting peizoelectric, the piezoelectric field pushes mobile charge carriers to opposite sides of the material. The mobile charges continue to migrate under the influence of the piezoelectric field until the enough mobile charge accumulates on opposite sides of the material to create an electric field which counters that of the piezoelectric and effectively screens the bulk of the material for the piezoelectric potential shift. 

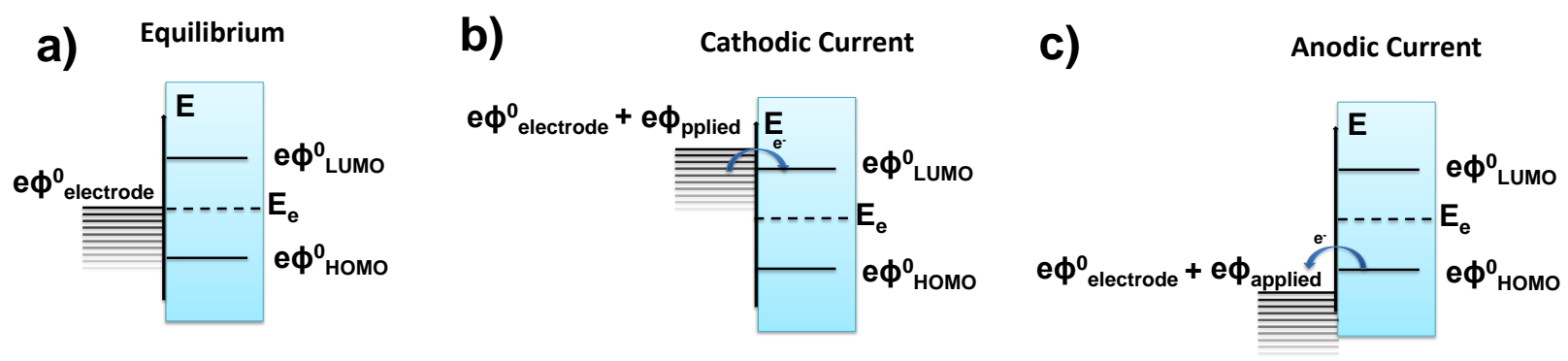

d)

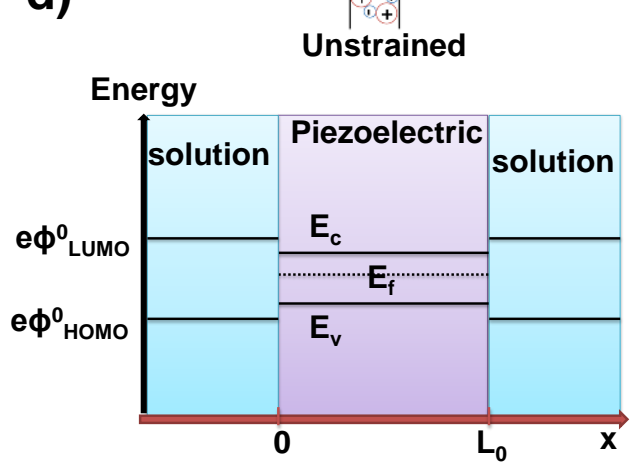

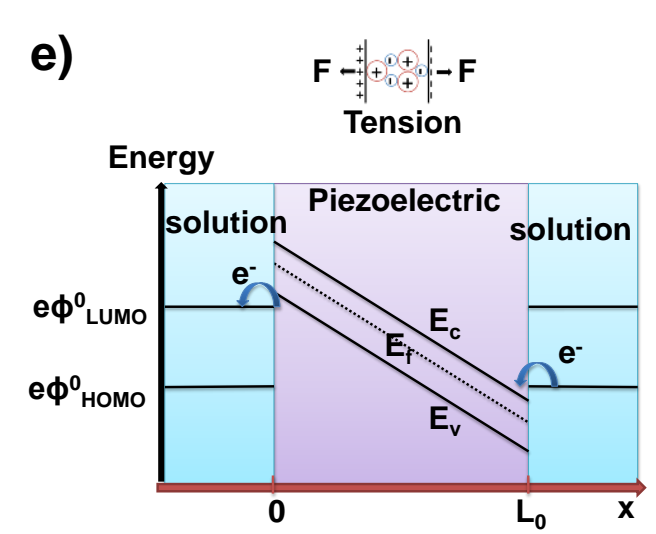

Figure 4. Changes in electrical potential between and electrode and solution and drive electrochemical reactions. a) A metal electrode is at equilibrium with its chemical surroundings. b) a potential is applied to the electrode, living up the energy of electrons in its orbitals above that of unoccupied states in solution. Electrons leave the electrode and go into solution, reducing the systems energy (cathodic current). c) a potential is applied to the electrode that lowers the energy of electrons in its orbitals below that of occupied states in solution. Electrons leave the solution and go into the electrode, reducing the systems energy (anodic current). d) A insulating piezoelectric is unstrained and in equilibrium with its chemical surroundings. e) The strained piezoelectric has dramatically changed the energy state of occupied and unoccupied states across the material, making electron transfer possible out of the valence band and into the conduction band. 


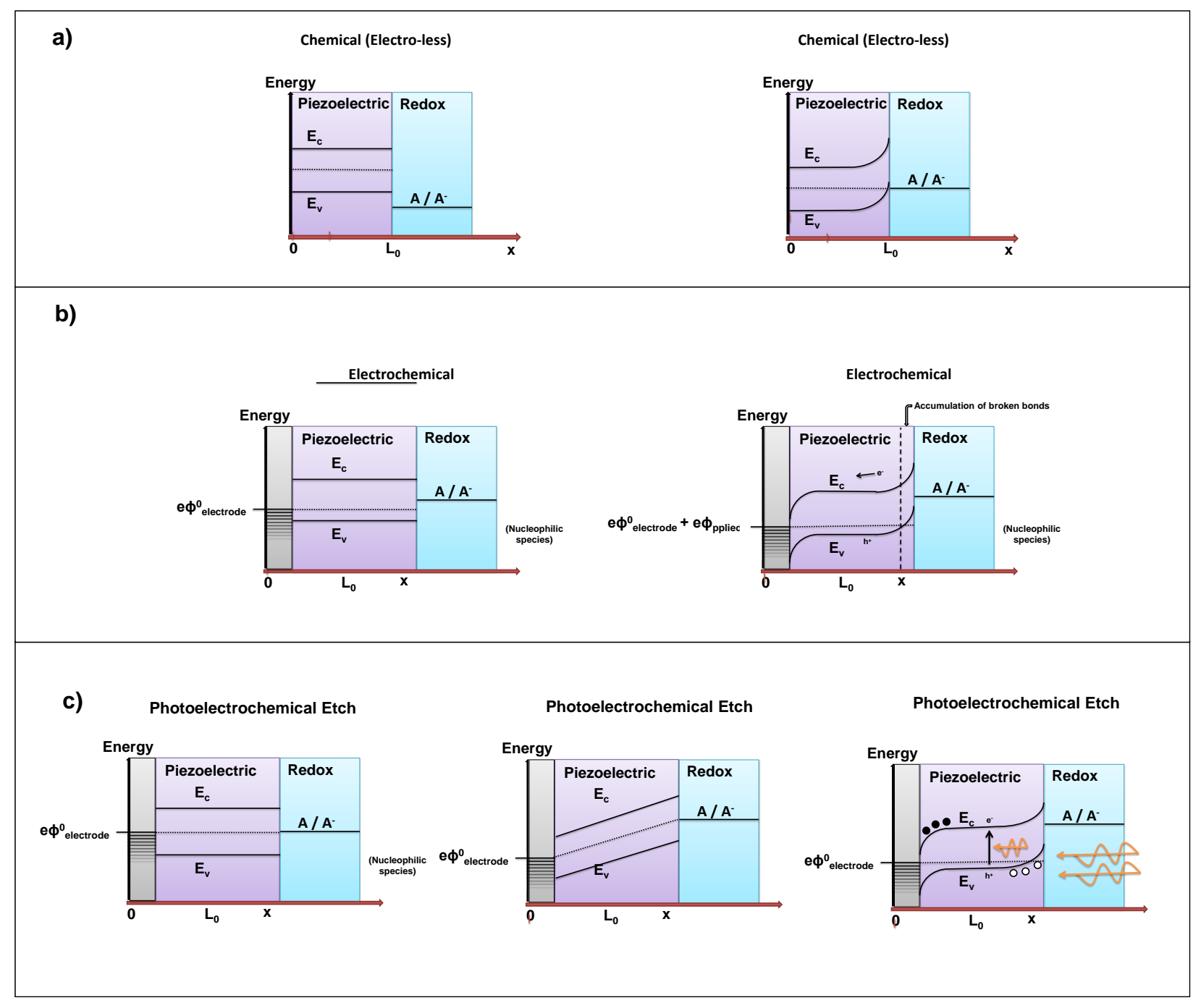

Figure 5. Etching can take place spontaneously or under an external bias. a) In pure chemical etching, the energetics of electroactive species in solution are naturally such that they are able to interface with the material, donating or extracting electrons as is necessary to isolate and extract atoms out form the surface and into solution. b) in electrochemical etching, and external bias is applied to a material, typically a metal or semiconductor, such that the energy of electronic states at the surface are made to be unoccupied (full of holes) and thus available for electroactive species in solution to donate to and interact with surface atoms. c) photoelectrochemical etching is typically used in insulating materials, where the application of a bias alone is insufficient to drive the electrochemical etching process. Applying a potential to an insulating material results in a relatively diffuse electric field permeating the material. This field may be insufficient to focus a high concentration of charge carriers at the material/solution interface. Photoexciting mobile charges within the material allows charge to migrate trhoughout the material, 
concentrating at the material's walls. As broken bonds accumulate at the material/solution interface, the surface atoms are made available for electron donation and interaction from electroactive species in solution.

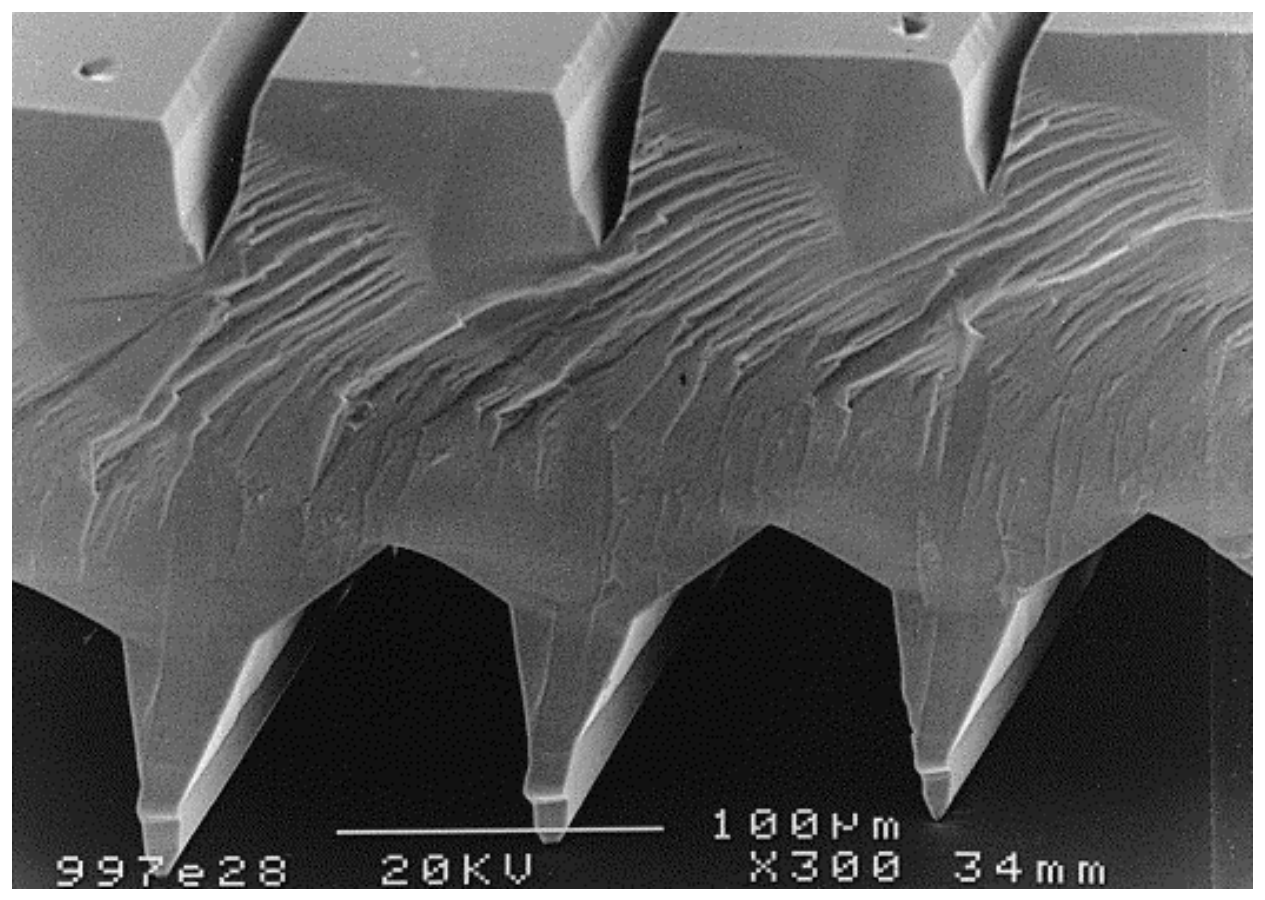

Figure 6. Etching rates of ferroelectrics depend on the local polarization. Here, a free-standing ferroelectric $\mathrm{LiNbO}_{3}$ is etched from both sides. Negative domains etch fasters than positive domains, resulting in this jagged structural formation. 

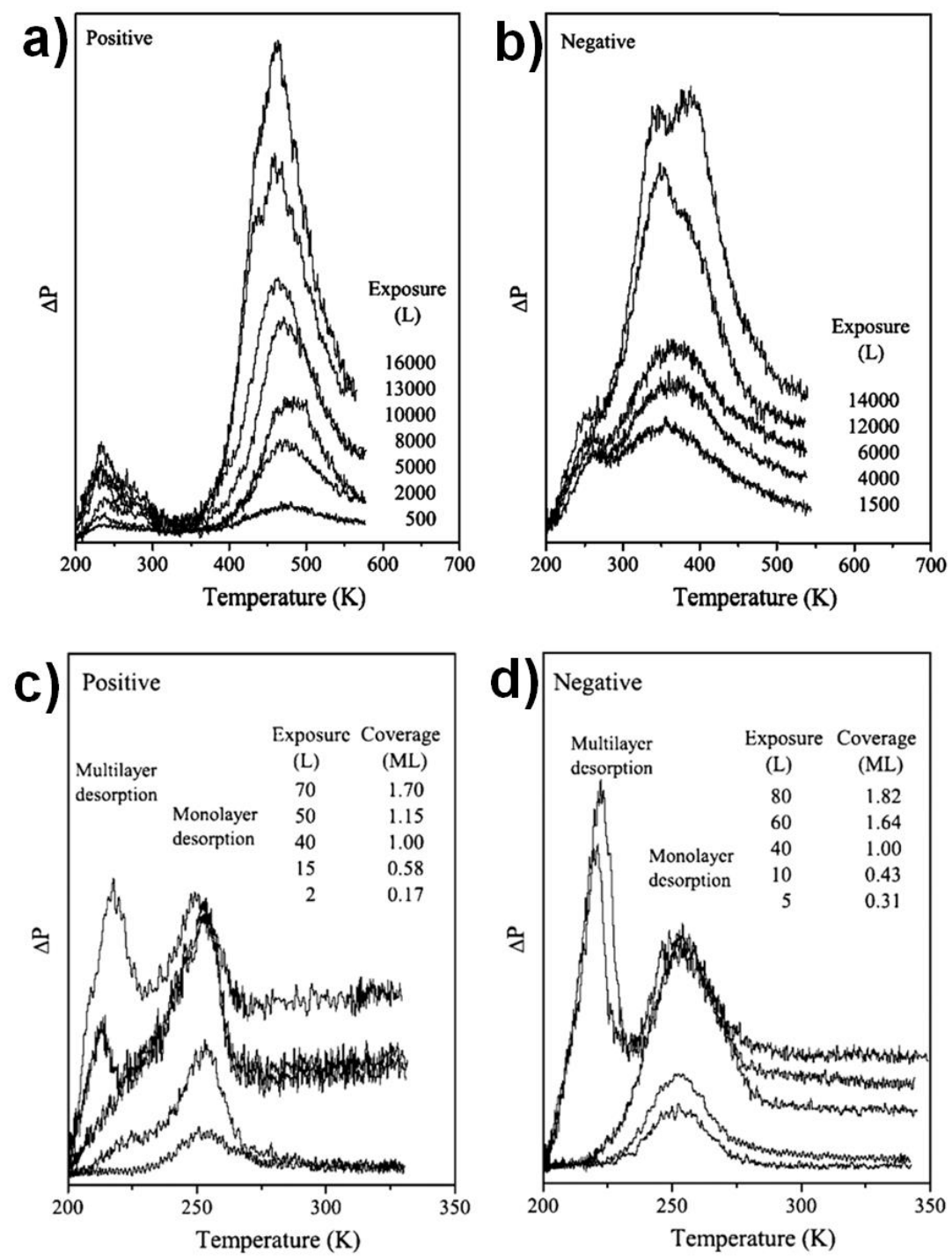

Figure 7. The bonding energy of surface absorbed molecules depends on both the polarization of the molecules and the surface. a) and b) are desorption curves for acedic acid on positively and negatively poled $\mathrm{LiNbO}_{3}(0001)$ surfaces, respectively. Acetic acid desorbs from both surfaces. The desorption peak for acedic acid on the positive face is $\sim 101 \mathrm{~K}$ higher than on the negative face, indicating that acetic acid adsorbs more strongly on the positive surface. c) and d) show that the desoroption peaks for the nonpolar dodecane are approximately equal. 

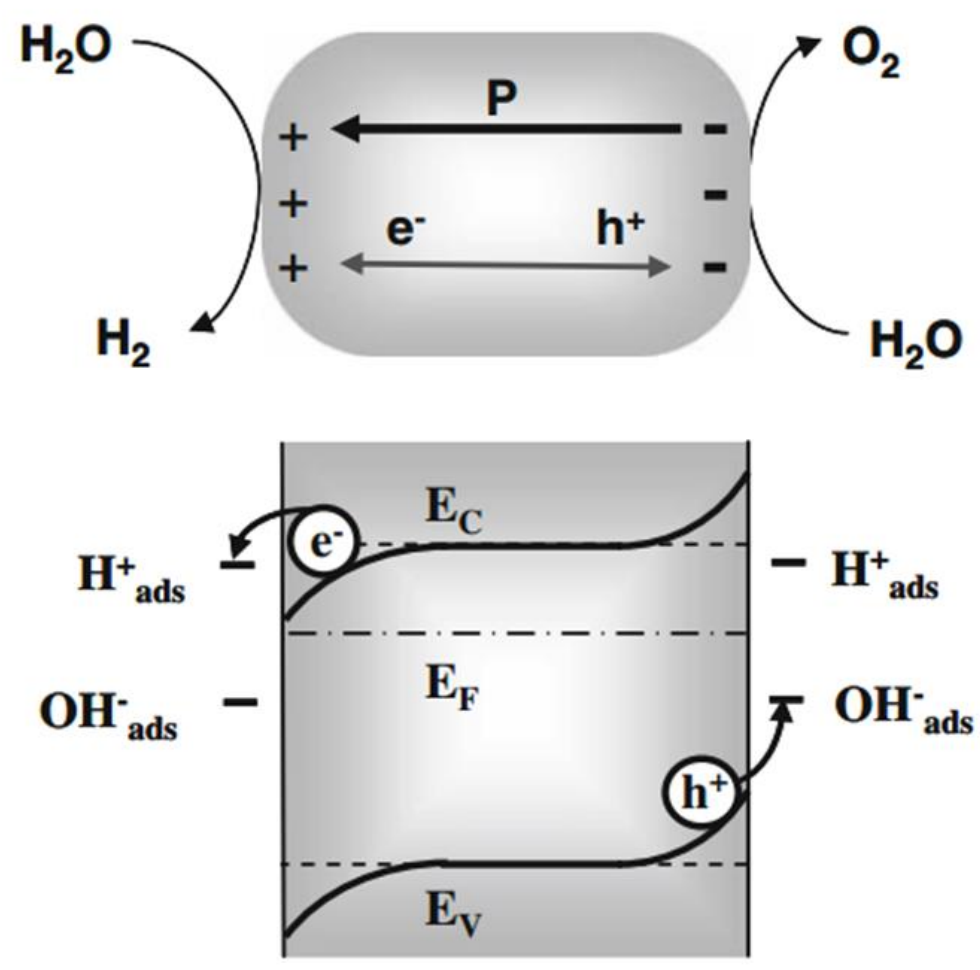

Figure 8. The spontaneous polarization in ferroelectric particles influences how mobile charges move about the particle, as well as the energetics of occupied and unoccupied states at the interface of the material and its surroundings. The polarization can dramatically effect how one end of the material reacts with its environment compared to the other. 

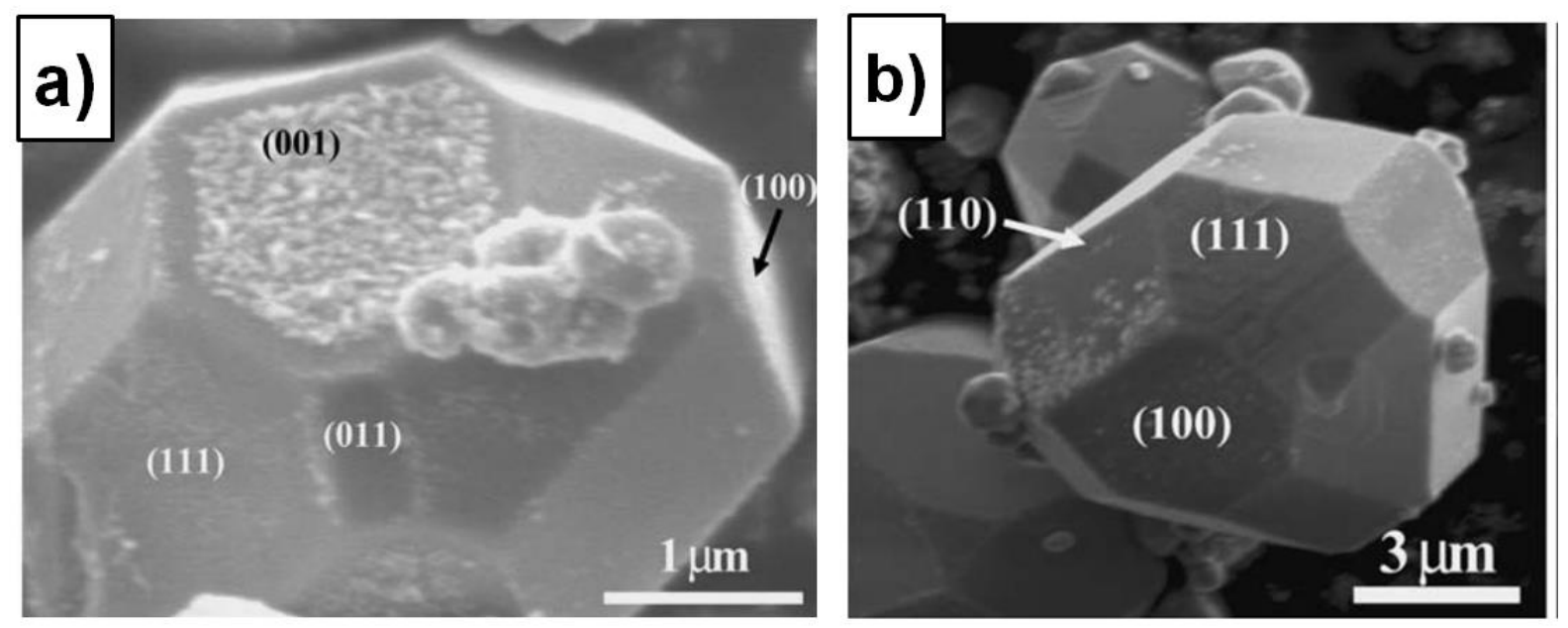

Figure 9. The effect of a single polarization within small ferroelectric particles can be seen in photodeposition experiments. a) an SEM of a ferroelectric $\mathrm{BaTiO}_{3}$ particle exposed to aqeous $\mathrm{AgNO}_{3}$ and photo-excited into reacting with its environment. The photodeposited silver deposits are primarily on the (001) facet. b) An SEM of a small $\mathrm{BaTiO}_{3}$ particle after it was exposed to aqueous $\mathrm{Pb}\left(\mathrm{C}_{2} \mathrm{H}_{3} \mathrm{O}_{2}\right)_{2}$ and driven by light to photo-react with its environment. The deposits seen in the picture contain oxidized lead and also primarily fall on a single facet, (100).
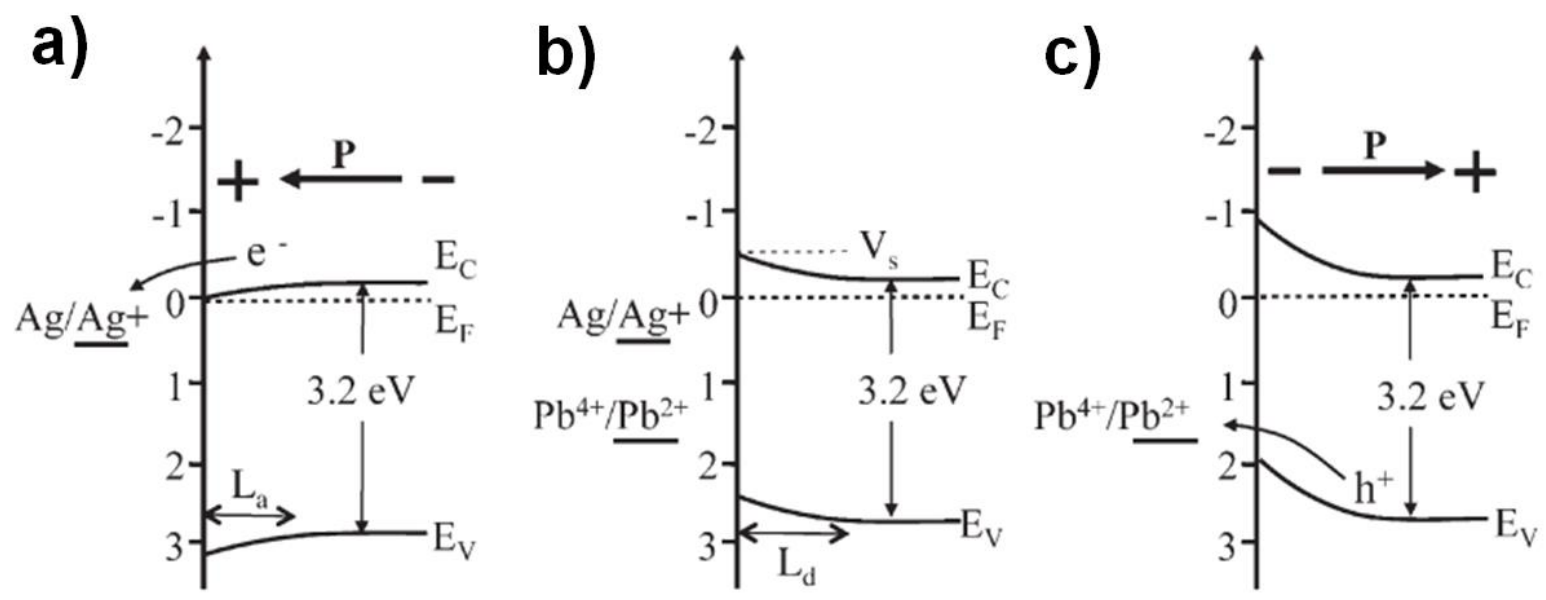

Figure 10. The spontaneous polarization of ferroelectric materials can effect what reactions take place on their surfaces. a) is an energy diagram depicting what the energy landscape looks like on a positive $\mathrm{BaTiO} 3$ domain. b) and c) depict the energetic landscape for a nonpolar and 
negative $\mathrm{BaTiO} 3$ domain, respectively. Both $\mathrm{Ag}^{+} / \mathrm{Ag}$ and $\mathrm{Pb}^{4+} / \mathrm{Pb}^{2+}$ are accessible redox potentials for BTO.
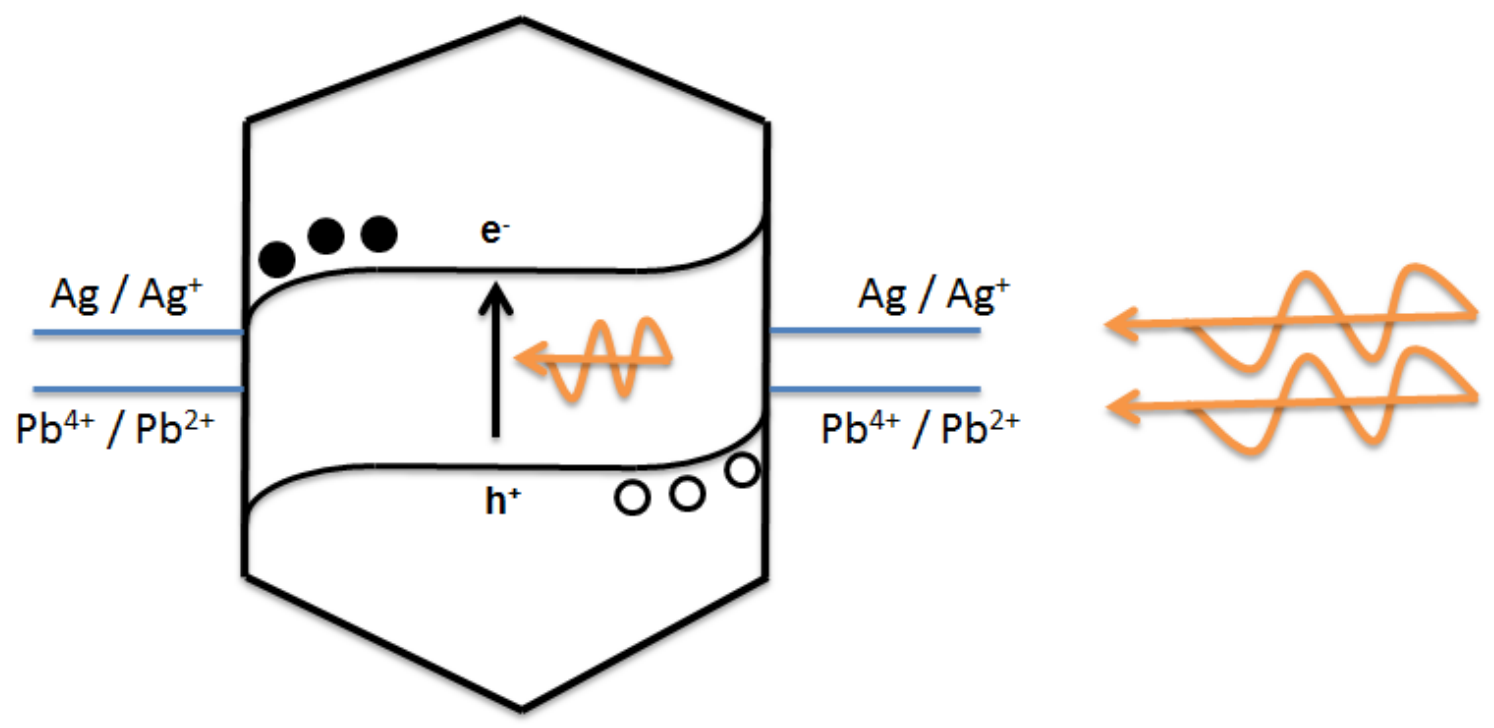

Figure 11. The spontaneous polarization of ferroelectric particles causes charge migration within the particles, resulting in reduction and oxidation reactions occurring on opposite sides of the material. 

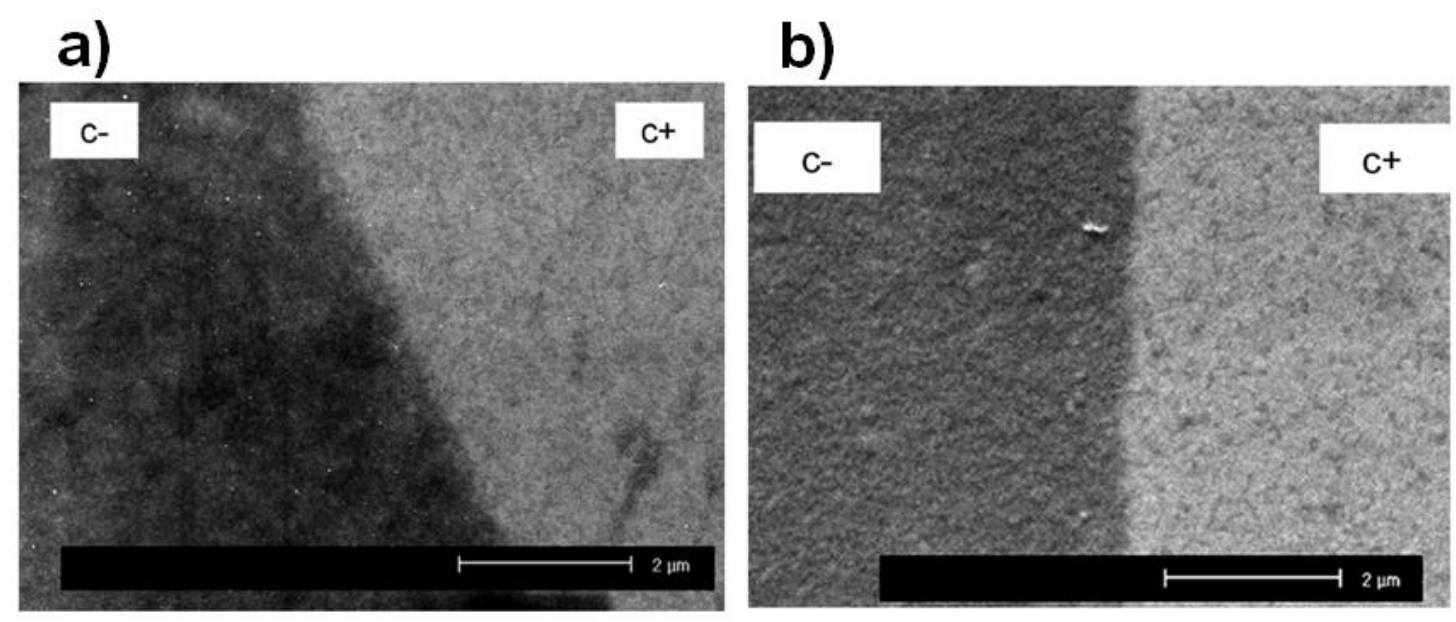

Figure 12. Ferroelectric domains dramatically effect the result of photodeposition experiments taking place on films. a) an SEM image of an interface between a negative and positive ferroelectric domain, after a photodeposition experiment. Sn deposited on the c+ domain from an aquous $\mathrm{SnCl}_{2}$ solution. The surface of the c- domain was unaffected by the photoexcitation. b) Fe deposited on $\mathrm{c}+$ in from an aquous $\mathrm{FeCl} 2$ solution. The surface of the c- domains remained unaffected in this sample 

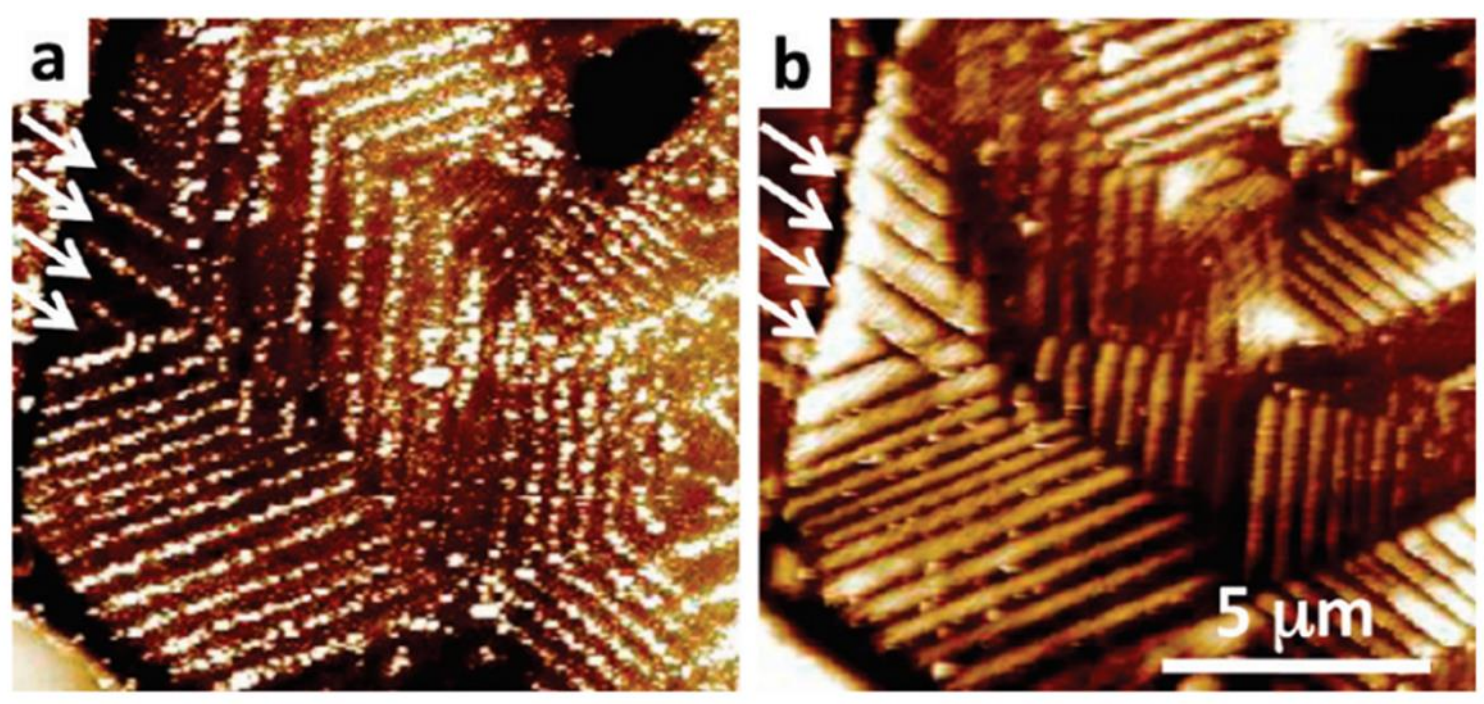

Figure 13. A $15 \mathrm{~nm}$ thick $\mathrm{TiO}_{2}$ coated, multi-domain ferroelectric substrate exposed to photodoposition experiments. a) a topographic AFM images of the film after reaction with aqueous $\mathrm{AgNO} 3$ solution. $\mathrm{Ag}$ is deposited on top of the $\mathrm{TiO}_{2}$ coating in the same pattern as the ferroelectric domains beneath the surface. b) A topographic AFM images of the film after reaction with a $\mathrm{Pb}$ solution. The arrows in figures a) and b) direct attention to domains that avoid deposition reactions under one experimental condition while encouraging it under another. 


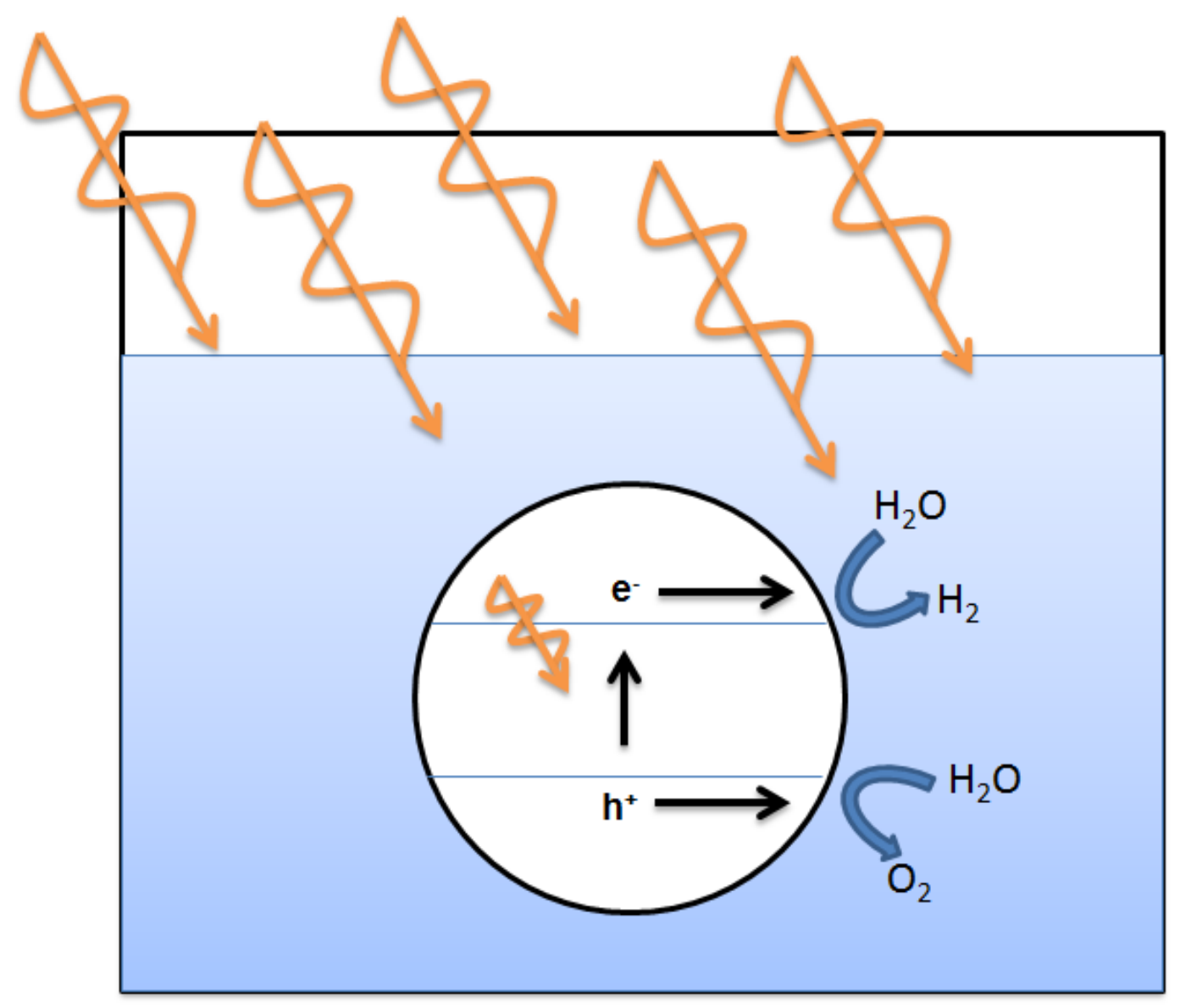

Figure 14. Free floating, semiconducting particles absorb light from the sun and use it to split water into $\mathrm{H}_{2}$ and $\mathrm{O}_{2}$ gas. 


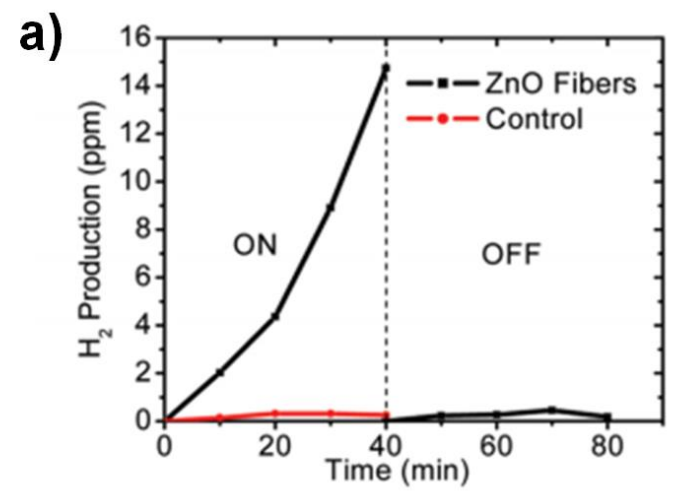

b)

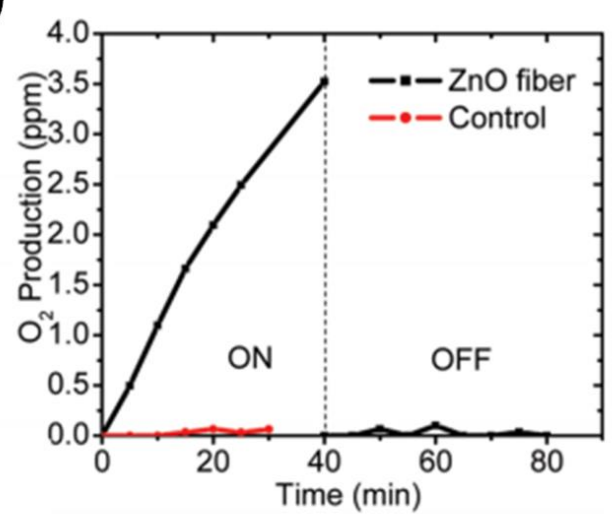

c)

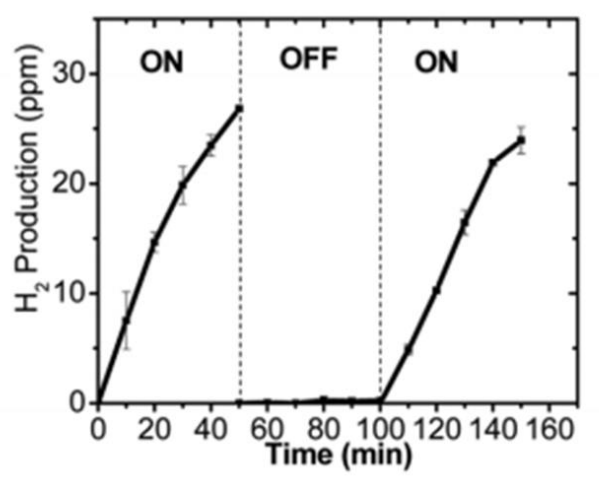

Figure 15. Ultrasonication of piezoelectric sires suspended in a water solution show an correlation with evolved $\mathrm{H}_{2}$ and $\mathrm{O}_{2}$ gas. A) and B) show the $\mathrm{H}_{2}$ and $\mathrm{O}_{2}$ production are both dependent on the piezoelectric nature of the material and the mechanical agitation to solution. $\mathrm{C}$ ) The system is repeatable. Here, where the environment is refreshed, the system continues to convert mechanical energy into $\mathrm{H}_{2}$ and $\mathrm{O}_{2}$ evolution. 

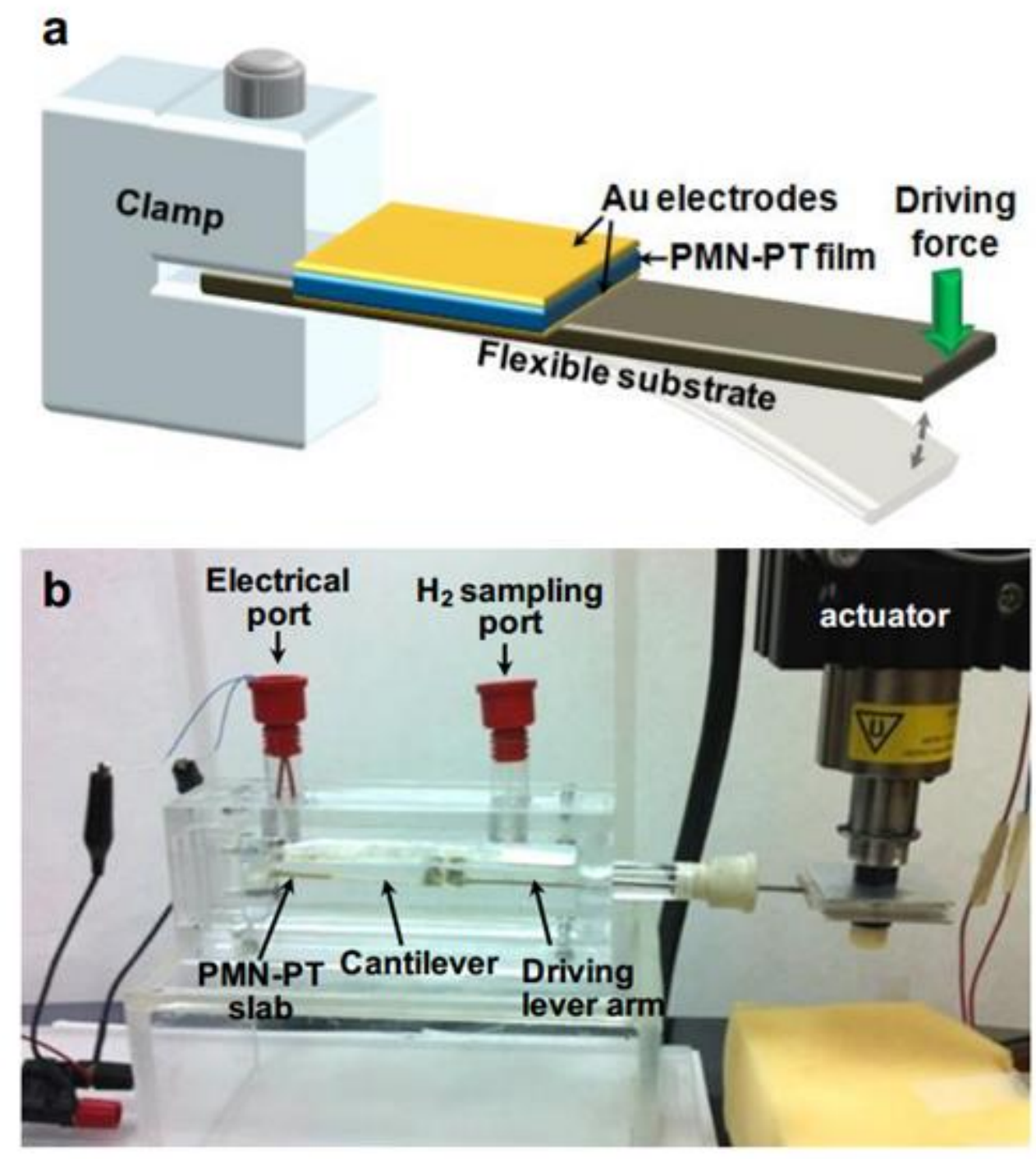

Figure 16. a) The piezoelectric cantilever setup for studying the piezocatalytic effect. It consists of a flexible substrate for strain induction, a piezoelectric element for generation of piezoelectric potential, and a clamp for securing the cantilever's base. b) Photo of the experimental setup and reaction apparatus used for studying piezocatalyzed hydrogen evolution from water. 

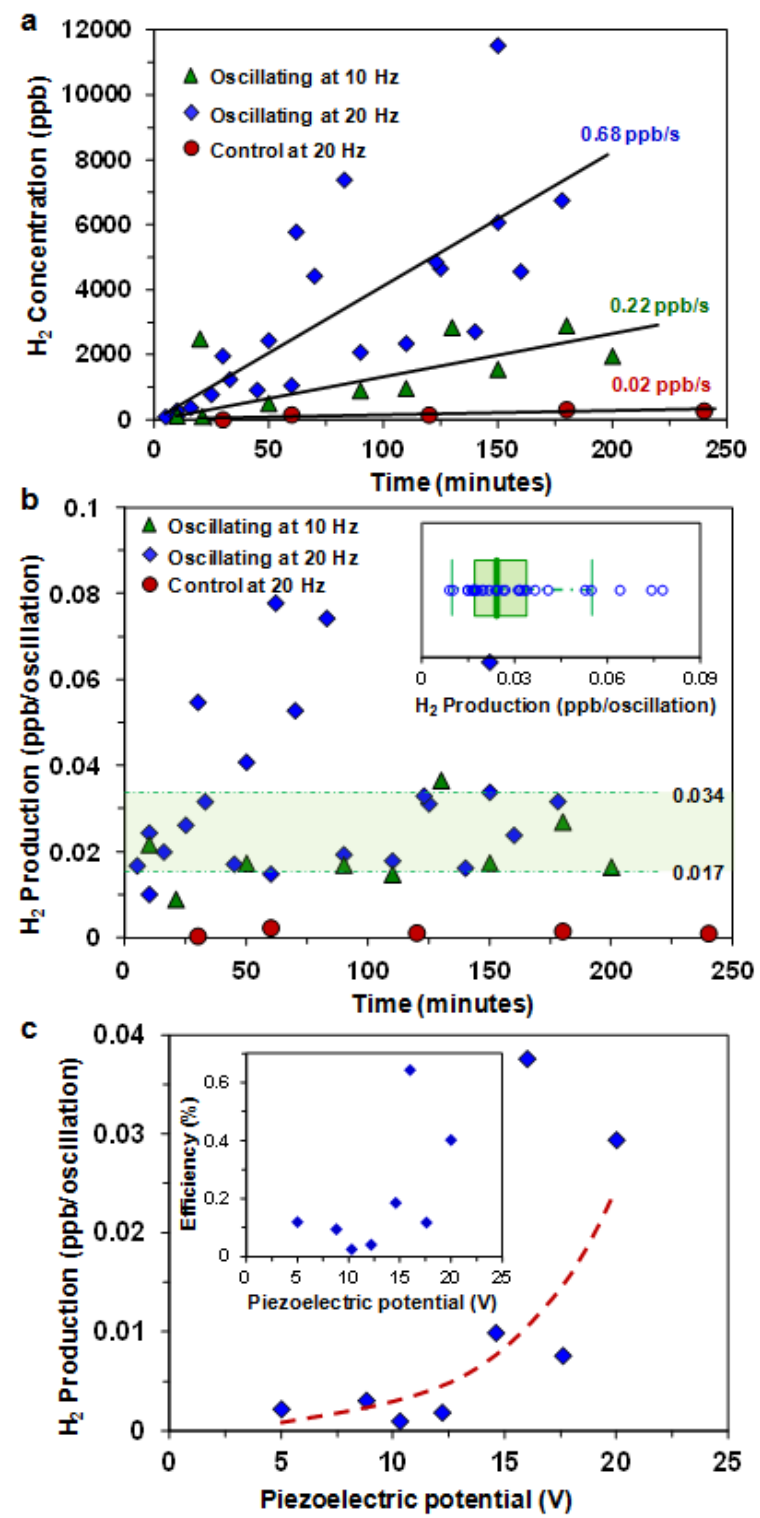

Figure 17. $\mathrm{H}_{2}$ evolution correlated with the direct piezoelectric effect. a) $\mathrm{H}_{2}$ concentrations measured as a function of oscillating time of the piezoelectric beam in DI water medium with a frequency of $10 \mathrm{~Hz}$ (green triangles) and $20 \mathrm{~Hz}$ (blue diamonds). A Si cantilever with identical configuration was used as a control and the corresponding $\mathrm{H}_{2}$ concentrations are marked by red circles. b) $\mathrm{H}_{2}$ production per oscillation values derived from experiments shown in a. Inset is a box-whisker plot of the data shown in $b$ representing a reference for the dispersion. c) $\mathrm{H}_{2}$ production per oscillation as a function of peak piezoelectric potential, where an exponential dependence was identified (red dashed line), consistent with the application of the Bulter-Volmer 
relationship. Inset is the corresponding peizocatalytic efficiency calculated as a function of peak piezoelectric potential.
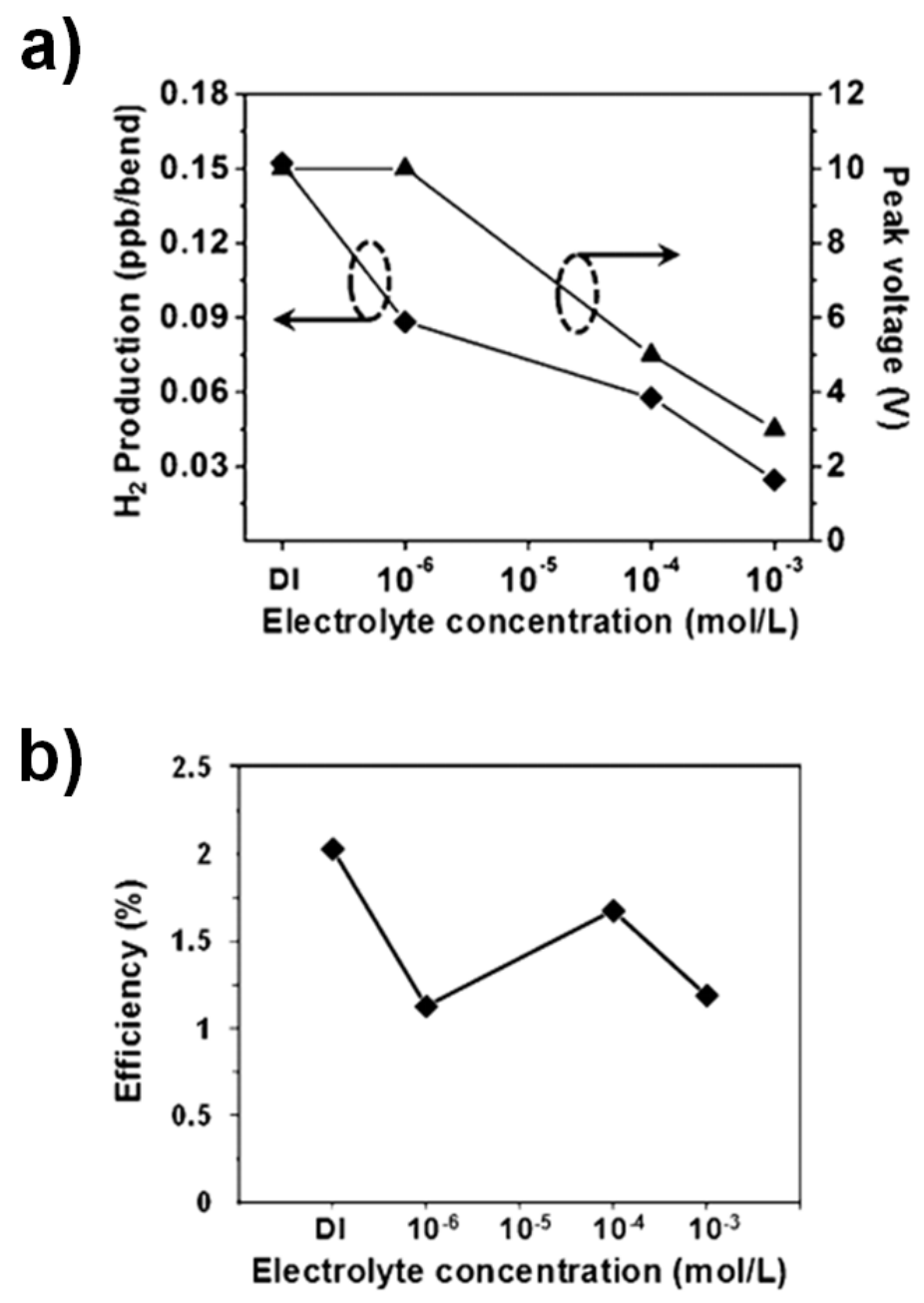

Figure 18. $\mathrm{H}_{2}$ evolution correlated with electrolyte concentration. a) The peak piezoelectric voltage (triangles) and $\mathrm{H}_{2}$ production rate (diamonds) as functions of the electrolyte concentration. b) Corresponding piezocatalytic efficiency of $\mathrm{H}_{2}$ production per strain as a function of electrolyte concentration. 


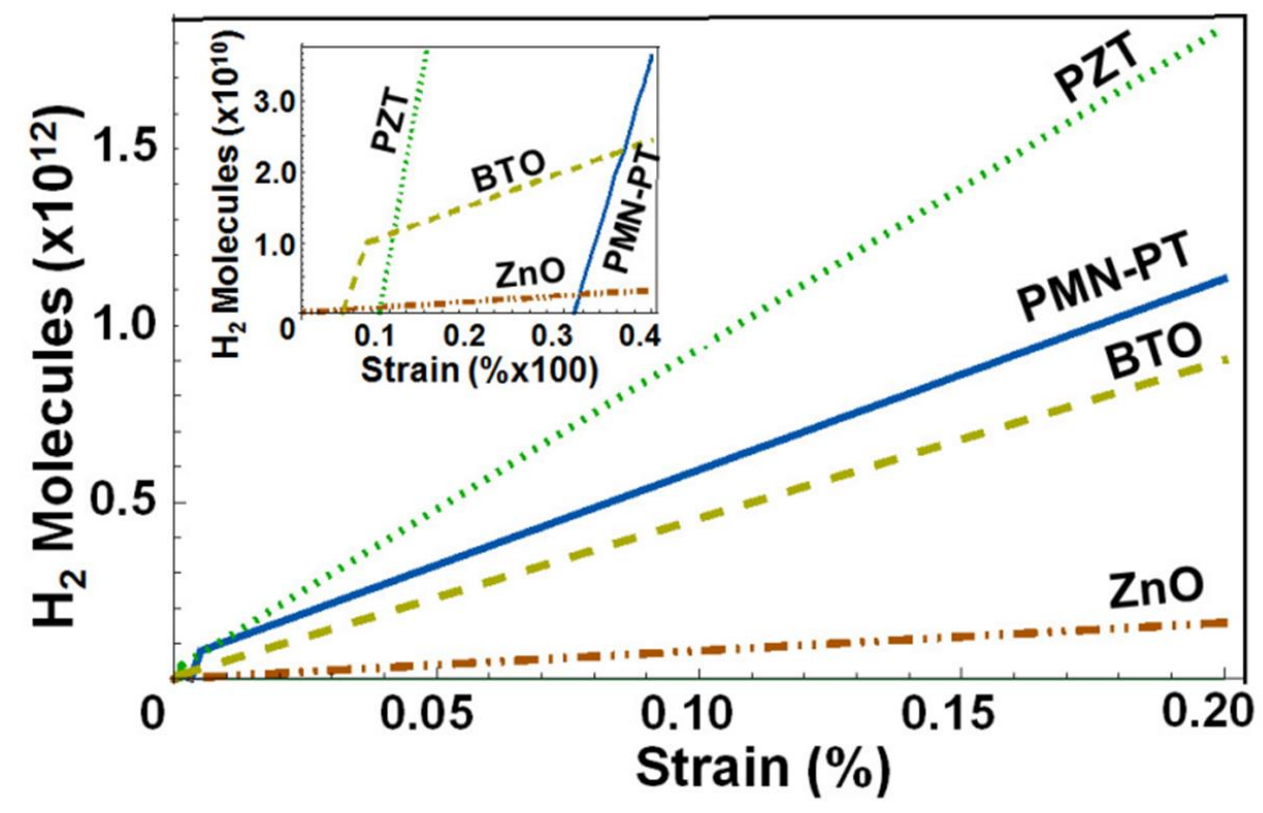

Figure 19. The $\mathrm{H}_{2}$ production per straining event predicted as a function of strain magnitude in the case of piezoelectric materials with metal electrodes on their surfaces. Inset shows the turnon strain value is dependent on the ratio of $\frac{\varepsilon_{r, x}}{\mathrm{~d}_{x k}}$. The electrical permittivity and surface charge density induced per unit strain is different from one material to the next, and thus the voltage change as a function of strain for each piezoelectric is different. These differences account of the difference in turn-on strain seen from one material to the next, as seen in the inset of the figure. 


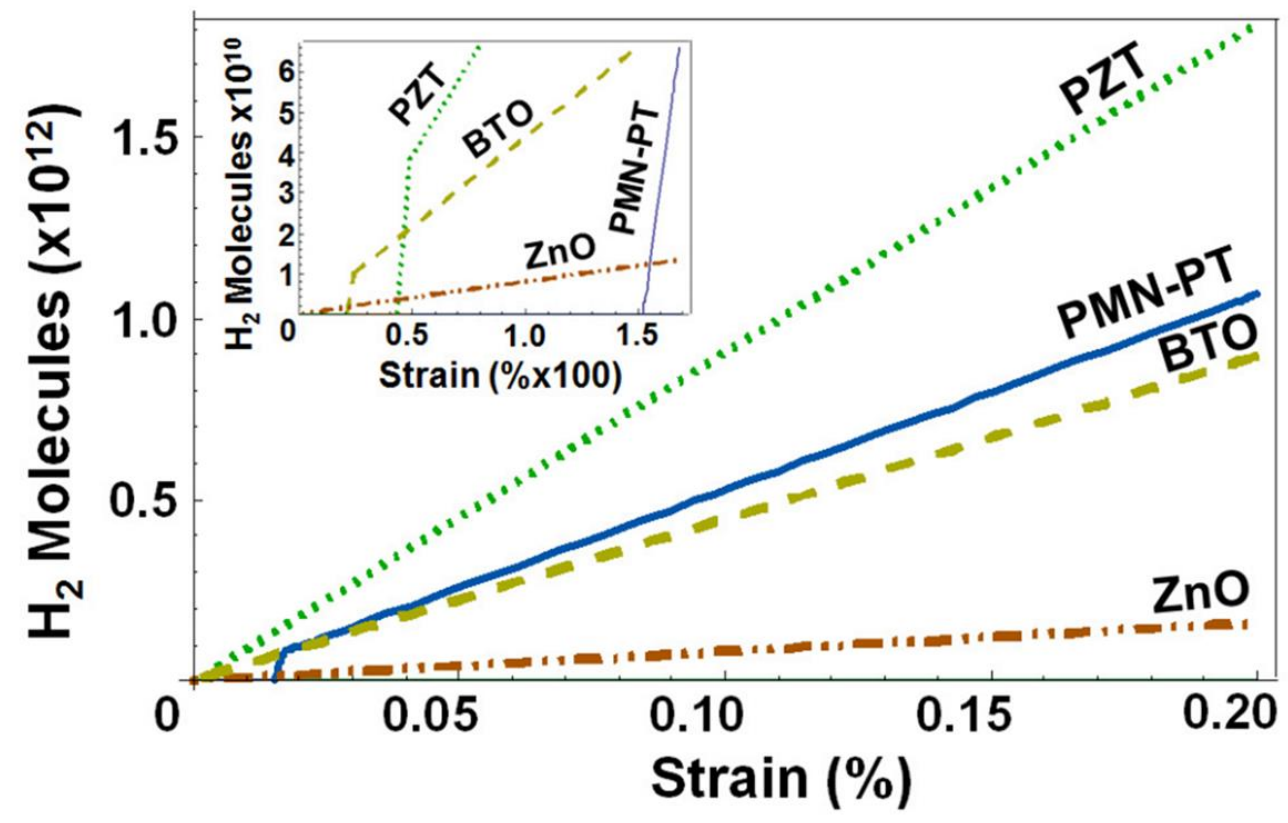

Figure 20. The $\mathrm{H}_{2}$ production per straining event predicted as a function of strain magnitude in the case of bare surfaced piezoelectric materials. Inset shows the turn-on strain values depends upon both the ratio of $\frac{\varepsilon_{r, x}}{\mathrm{~d}_{x k}}$ and the value of $\varphi_{O_{p}}$. The turn-on strain for bare surfaced piezoelectrics is different from their metal coated counterparts. Shown here, all turn-on strains were larger than when the piezoelectrics have metal coatings, but that need not always be the case. At high strain values, the hydrogen production per unit strain is equivalent to the scenario where the piezoelectrics have metal coatings because the valence band states in the materials are taken as continuum. 

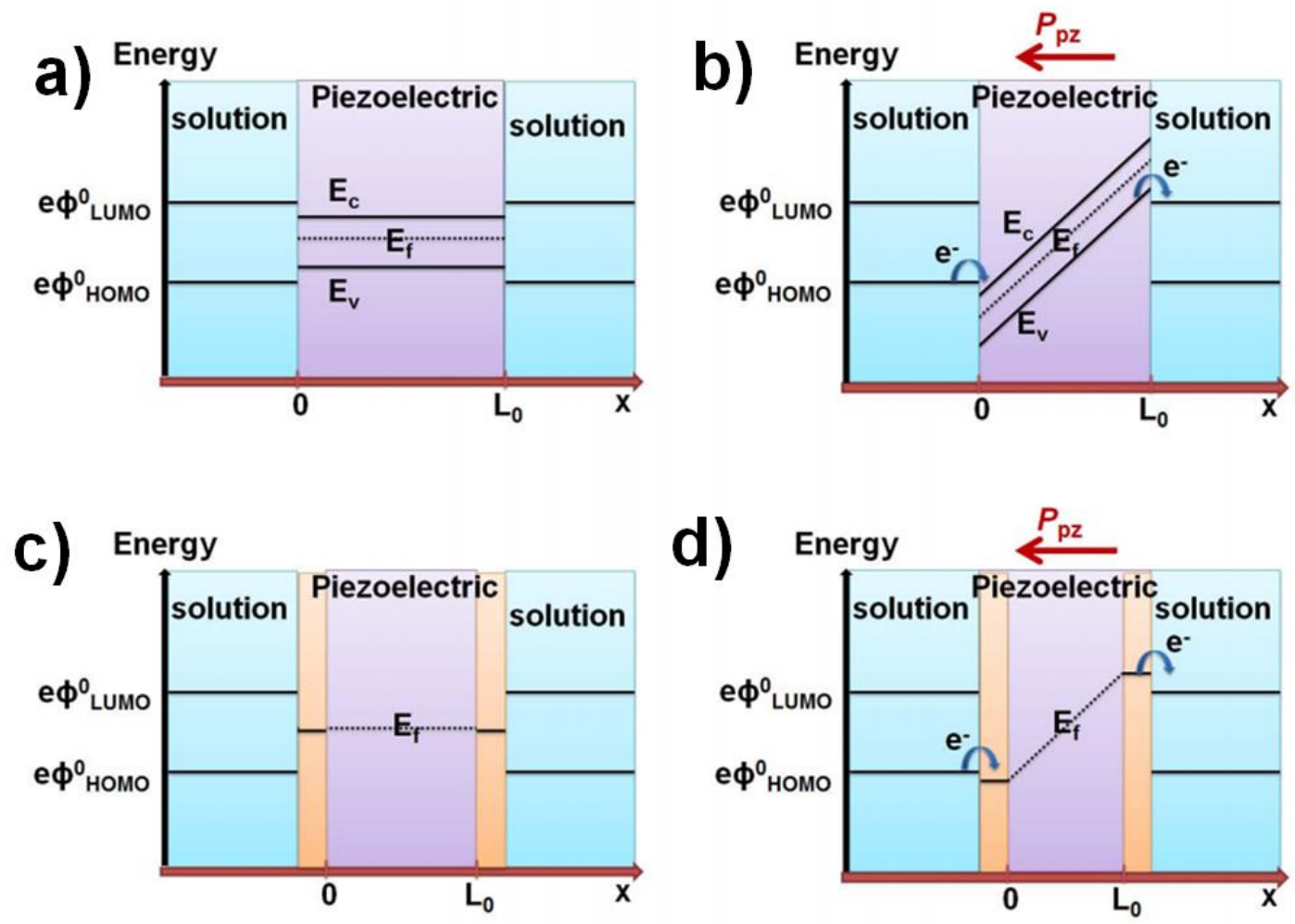

Figure 21. Energy diagrams describing the electrochemistry and piezocatalysis process. a) and b) In the case of a bare piezoelectric, conduction and valence bands act as the reservoirs for electrons donated or accepted from molecules in solution. The piezoelectric polarization applies a variable bias across the material, lifting and lowering valence band and conduction band energies. c) and d) Applying electrodes to the piezoelectric simplifies the electron reservoir to that of the metal's Fermi energy, while maintaining the piezoelectric potential as the source of bias. 


\section{References:}

1. Damjanovic, D. Reports on Progress in Physics 61 (1998) 1267-1324.

2. Bowen, C. R.; Kim, H. A.; Weaver, P. M.; Dunn, S. Energy \& Environmental Science 7 (2014) 25-44.

3. Wang, Z. L. Nano Today 5 (2010) 540-552.

4. Wang, Z. L. Advanced Materials 24 (2012) 4632-4646.

5. $\quad$ Zhang, Y.; Liu, Y.; Wang, Z. L. Advanced Materials 23 (2011) 3004-3013.

6. $\quad$ Starr, M. B.; Shi, J.; Wang, X. D. Angewandte Chemie-International Edition 51 (2012) 5962-5966.

7. Starr, M. B.; Wang, X. D. Scientific Reports 3 (2013) 2160.

8. Gao, Z. D.; Wang, Q. J.; Zhang, Y.; Zhu, S. N. Optical Materials 30 (2008) 847-850.

9. Hiranaga, Y.; Wagatsuma, Y.; Cho, Y. Japanese Journal of Applied Physics Part 2-Letters \& Express Letters 43 (2004) L569-L571.

10. Kobayashi, T.; Ichiki, M.; Kondou, R.; Nakamura, K.; Maeda, R. Journal of Micromechanics and Microengineering 18 (2008) 035007.

11. Lee, Y. J.; Subramania, G.; Clem, P. G., Wet etching of sol-gel deposited lead lanthanum zirconate titanate for photonic crystal applications - art. no. 612814. In Photonic Crystal Materials and Devices IV, Adibi, A.; Lin, S. Y.; Scherer, A., Eds. 2006; Vol. 6128, pp 12814-12814.

12. Park, J. H.; Kim, H. H.; Jang, N. W.; Song, Y. J.; Joo, H. J.; Kang, H. Y.; Lee, S. Y.; Kim, K. Integrated Ferroelectrics 53 (2003) 307-315.

13. Petraru, A.; Schubert, J.; Schmid, M.; Buchal, C. Applied Physics Letters 81 (2002) 1375-1377.

14. Sun, D. G.; Liu, Z. F.; Huang, Y. Y.; Ho, S. T.; Towner, D. J.; Wessels, B. W. Optics Communications 255 (2005) 319-330.

15. Tyholdt, F.; Dorey, R. A.; Raeder, H. Journal of Electroceramics 19 (2007) 315-319.

16. Wessels, B. W. Journal of Electroceramics 13 (2004) 135-138.

17. Wessels, B. W., Ferroelectric epitaxial thin films for integrated optics. In Annual Review of Materials Research, 2007; Vol. 37, pp 659-679.

18. Yeh, T. H.; Suresh, M. B.; Shen, J. N.; Yu, J. C.; Chou, C. C. Ferroelectrics 380 (2009) 97-101.

19. Yun, Y.; Altman, E. I. Journal of the American Chemical Society 129 (2007) 15684-15689.

20. Dubey, A. K.; Yamada, H.; Kakimoto, K.-i. Journal of Crystal Growth 382 (2013) 7-14.

21. Bhardwaj, A.; Burbure, N. V.; Gamalski, A.; Rohrer, G. S. Chemistry of Materials 22 (2010) 35273534.

22. Bhardwaj, A.; Burbure, N. V.; Rohrer, G. S. Journal of the American Ceramic Society 93 (2010) 4129-4134.

23. Jones, P. M.; Gallardo, D. E.; Dunn, S. Chemistry of Materials 20 (2008) 5901-5906.

24. Kim, J. H.; Kwon, S.; Yang, W. Journal of Nanoscience and Nanotechnology 14 (2014) 7985-7989.

25. Schultz, A. M.; Zhang, Y. L.; Salvador, P. A.; Rohrer, G. S. Acs Applied Materials \& Interfaces 3 (2011) 1562-1567.

26. Tiwari, D.; Dunn, S.; Zhang, Q. Materials Research Bulletin 44 (2009) 1219-1224.

27. Zhang, M.; Jiang, C. X.; Dong, W.; Zheng, F. G.; Fang, L.; Su, X. D.; Shen, M. R. Applied Physics Letters 103 (2013).

28. Zhen, C.; Yu, J. C.; Liu, G.; Cheng, H. M. Chemical Communications 50 (2014) 10416-10419.

29. Hong, K. S.; Xu, H. F.; Konishi, H.; Li, X. C. Journal of Physical Chemistry Letters 1 (2010) 997-1002.

30. Hong, K. S.; Xu, H. F.; Konishi, H.; Li, X. C. Journal of Physical Chemistry C 116 (2012) 1304513051.

31. Ebrahimi, H.; Yaghoubi, H.; Giammattei, F.; Takshi, A. Electrochimica Acta 134 (2014) 435-441.

32. Baborowski, J. Journal of Electroceramics 12 (2004) 33-51.

33. Eom, C. B.; Trolier-McKinstry, S. Mrs Bulletin 37 (2012) 1007-1021.

34. Muralt, P. Journal of the American Ceramic Society 91 (2008) 1385-1396. 
35. Polla, D. L.; Francis, L. F. Annual Review of Materials Science 28 (1998) 563-597.

36. Polla, D. L.; Schiller, P. J. Integrated Ferroelectrics 7 (1995) 359-370.

37. Tadigadapa, S.; Mateti, K. Measurement Science \& Technology 20 (2009).

38. Trolier-McKinstry, S.; Muralt, P. Journal of Electroceramics 12 (2004) 7-17.

39. Varadan, V. K.; Varadan, V. V. Smart Materials \& Structures 9 (2000) 953-972.

40. Xu, B. M.; Cross, L. E.; Bernstein, J. J. Thin Solid Films 377 (2000) 712-718.

41. Cimalla, V.; Pezoldt, J.; Ambacher, O. Journal of Physics D-Applied Physics 40 (2007) 6386-6434.

42. Dalton, D.; Gnadinger, F.; Klingensmith, D.; Olariu, V.; Kalkur, T.; Rahman, M.; Mahmud, A. Integrated Ferroelectrics 81 (2006) 187-196.

43. Wang, J.; Ju, S.; Li, Z. Y. Journal of Physics D-Applied Physics 43 (2010).

44. Zenkevich, A.; Minnekaev, M.; Matveyev, Y.; Lebedinskii, Y.; Bulakh, K.; Chouprik, A.; Baturin, A.; Maksimova, K.; Thiess, S.; Drube, W. Applied Physics Letters 102 (2013).

45. Bermudez, V.; Caccavale, F.; Sada, C.; Segato, F.; Dieguez, E. Journal of Crystal Growth 191 (1998) 589-593.

46. Holstein, W. L. Journal of Crystal Growth 171 (1997) 477-484.

47. Jung, D. J.; Morrison, F. D.; Dawber, M.; Kim, H. H.; Kim, K.; Scott, J. F. Journal of Applied Physics 95 (2004) 4968-4975.

48. Kubel, F.; Schmid, H. Journal of Crystal Growth 129 (1993) 515-524.

49. Liu, X. Y.; Terabe, K.; Nakamura, M.; Takekawa, S.; Kitamura, K. Journal of Applied Physics 97 (2005).

50. $\quad$ Nassau, K.; Levinste.Hj; Loiacono, G. M. Applied Physics Letters 6 (1965) 228-\&.

51. Nassau, K.; Levinste.Hj; Loiacono, G. M. Journal of Physics and Chemistry of Solids 27 (1966) 983-

$\&$.

52. Sones, C. L.; Mailis, S.; Brocklesby, W. S.; Eason, R. W.; Owen, J. R. Journal of Materials Chemistry 12 (2002) 295-298.

53. Garra, J.; Vohs, J. M.; Bonnell, D. A. Surface Science 603 (2009) 1106-1114.

54. Ramos-Moore, E.; Lederman, D.; Cabrera, A. L. Applied Surface Science 258 (2011) 1181-1183.

55. Stoflea, L. E.; Apostol, N. G.; Trupina, L.; Teodorescu, C. M. Journal of Materials Chemistry A 2 (2014) 14386-14392.

56. Zhang, Z. Z.; Sharma, P.; Borca, C. N.; Dowben, P. A.; Gruverman, A. Applied Physics Letters 97 (2010).

57. Zhao, M. H.; Bonnell, D. A.; Vohs, J. M. Surface Science 602 (2008) 2849-2855.

58. Habicht, S.; Nemanich, R. J.; Gruverman, A. Nanotechnology 19 (2008).

59. Giocondi, J. L.; Rohrer, G. S. Topics in Catalysis 49 (2008) 18-23.

60. Shi, J.; Starr, M. B.; Wang, X. D. Advanced Materials 24 (2012) 4683-4691.

61. Burbure, N. V.; Salvador, P. A.; Rohrer, G. S. Chemistry of Materials 22 (2010) 5823-5830.

62. Zhang, Y. L.; Schultz, A. M.; Salvador, P. A.; Rohrer, G. S. Journal of Materials Chemistry 21 (2011) 4168-4174.

63. Alstrum-Acevedo, J. H.; Brennaman, M. K.; Meyer, T. J. Inorganic Chemistry 44 (2005) 6802-6827.

64. Gust, D.; Moore, T. A.; Moore, A. L. Accounts of Chemical Research 42 (2009) 1890-1898.

65. Lewis, N. S.; Nocera, D. G. Proceedings of the National Academy of Sciences of the United States of America 103 (2006) 15729-15735.

66. Maeda, K.; Domen, K. Journal of Physical Chemistry Letters 1 (2010) 2655-2661.

67. Roy, S. C.; Varghese, O. K.; Paulose, M.; Grimes, C. A. Acs Nano 4 (2010) 1259-1278.

68. Varghese, O. K.; Paulose, M.; LaTempa, T. J.; Grimes, C. A. Nano Letters 9 (2009) 731-737.

69. Zou, Z. G.; Ye, J. H.; Sayama, K.; Arakawa, H. Nature 414 (2001) 625-627.

70. Chen, X. B.; Shen, S. H.; Guo, L. J.; Mao, S. S. Chemical Reviews 110 (2010) 6503-6570. 
71. Hara, M.; Kondo, T.; Komoda, M.; Ikeda, S.; Shinohara, K.; Tanaka, A.; Kondo, J. N.; Domen, K. Chemical Communications (1998) 357-358.

72. Hernandez-Alonso, M. D.; Fresno, F.; Suarez, S.; Coronado, J. M. Energy \& Environmental Science 2 (2009) 1231-1257.

73. Kato, H.; Asakura, K.; Kudo, A. Journal of the American Chemical Society 125 (2003) 3082-3089.

74. Kato, H.; Kudo, A. Journal of Physical Chemistry B 105 (2001) 4285-4292.

75. Kitano, M.; Matsuoka, M.; Ueshima, M.; Anpo, M. Applied Catalysis a-General 325 (2007) 1-14.

76. Kudo, A.; Miseki, Y. Chemical Society Reviews 38 (2009) 253-278.

77. Li, Q.; Guo, B. D.; Yu, J. G.; Ran, J. R.; Zhang, B. H.; Yan, H. J.; Gong, J. R. Journal of the American Chemical Society 133 (2011) 10878-10884.

78. Liu, G.; Wang, L. Z.; Yang, H. G.; Cheng, H. M.; Lu, G. Q. Journal of Materials Chemistry 20 (2010) 831-843.

79. Liu, G.; Yang, H. G.; Wang, X. W.; Cheng, L. N.; Pan, J.; Lu, G. Q.; Cheng, H. M. Journal of the American Chemical Society 131 (2009) 12868-+.

80. Luo, H. M.; Takata, T.; Lee, Y. G.; Zhao, J. F.; Domen, K.; Yan, Y. S. Chemistry of Materials 16 (2004) 846-849.

81. Ng, Y. H.; Iwase, A.; Kudo, A.; Amal, R. Journal of Physical Chemistry Letters 1 (2010) 2607-2612.

82. Ni, M.; Leung, M. K. H.; Leung, D. Y. C.; Sumathy, K. Renewable \& Sustainable Energy Reviews 11 (2007) 401-425.

83. Osterloh, F. E. Chemistry of Materials 20 (2008) 35-54.

84. Park, J. H.; Kim, S.; Bard, A. J. Nano Letters 6 (2006) 24-28.

85. Takata, T.; Furumi, Y.; Shinohara, K.; Tanaka, A.; Hara, M.; Kondo, J. N.; Domen, K. Chemistry of Materials 9 (1997) 1063-\&.

86. Youngblood, W. J.; Lee, S. H. A.; Maeda, K.; Mallouk, T. E. Accounts of Chemical Research 42 (2009) 1966-1973.

87. Yu, J. G.; Qi, L. F.; Jaroniec, M. Journal of Physical Chemistry C 114 (2010) 13118-13125.

88. Zhang, X. Y.; Li, H. P.; Cui, X. L.; Lin, Y. H. Journal of Materials Chemistry 20 (2010) 2801-2806.

89. Fan, H. M.; Li, H. Y.; Liu, B. K.; Lu, Y. C.; Xie, T. F.; Wang, D. J. Acs Applied Materials \& Interfaces 4 (2012) 4853-4857.

90. Kohno, M.; Kaneko, T.; Ogura, S.; Sato, K.; Inoue, Y. Journal of the Chemical Society-Faraday Transactions 94 (1998) 89-94.

91. Lin, X.; Guan, Q. F.; Li, H. B.; Ba, C. H.; Wang, D. D.; Meng, F. J. Chinese Journal of Inorganic Chemistry 28 (2012) 2248-2256.

92. Maeda, K. Acs Applied Materials \& Interfaces 6 (2014) 2167-2173.

93. Ren, P. R.; Fan, H. Q.; Wang, X. Applied Physics a-Materials Science \& Processing 111 (2013) 1139-1145.

94. Stanca, S. E.; Muller, R.; Urban, M.; Csaki, A.; Froehlich, F.; Krafft, C.; Popp, J.; Fritzsche, W. Catalysis Science \& Technology 2 (2012) 1472-1479.

95. Shi, J.; Starr, M. B.; Xiang, H.; Hara, Y.; Anderson, M. A.; Seo, J. H.; Ma, Z. Q.; Wang, X. D. Nano Letters 11 (2011) 5587-5593. 


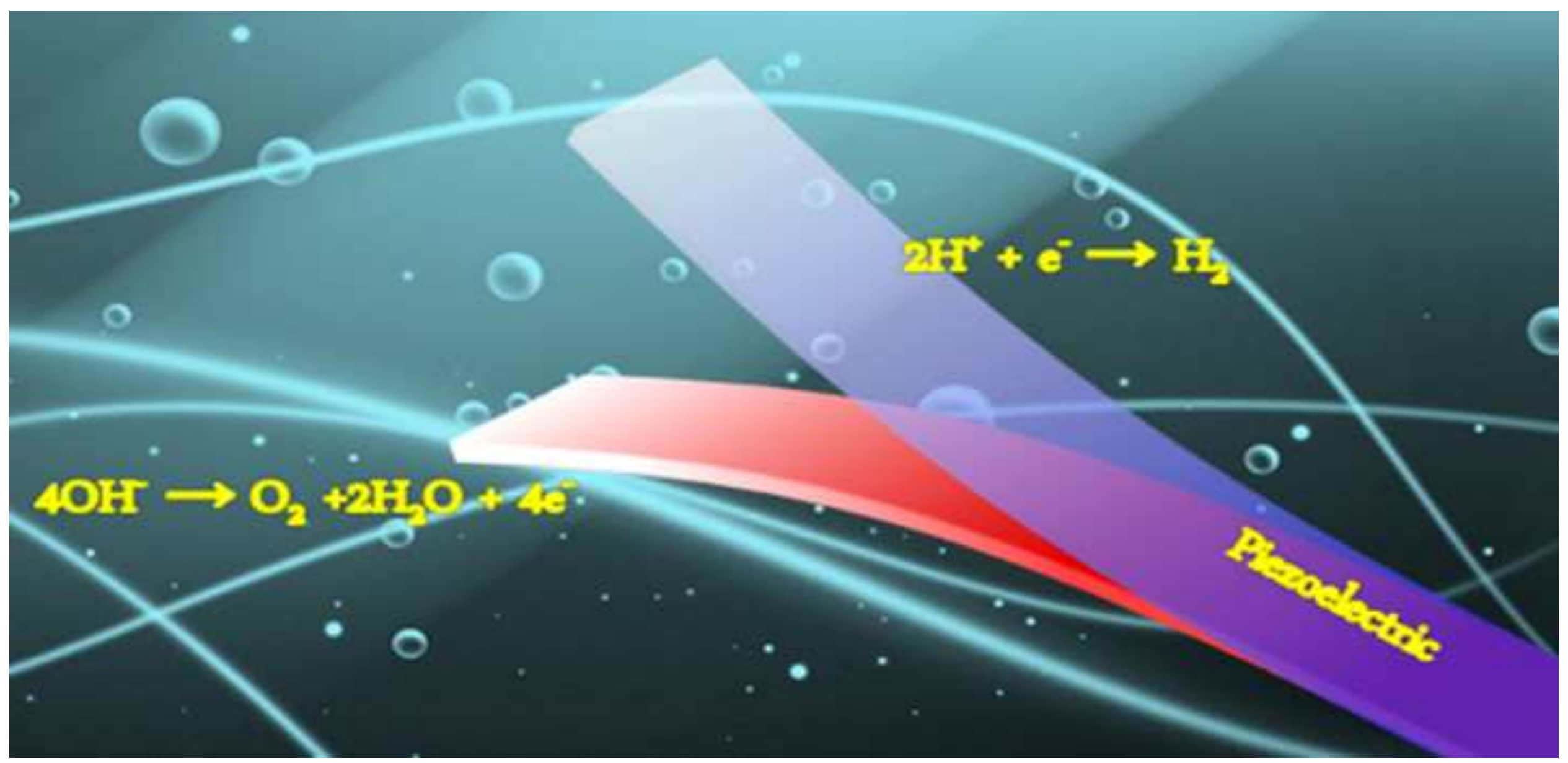

